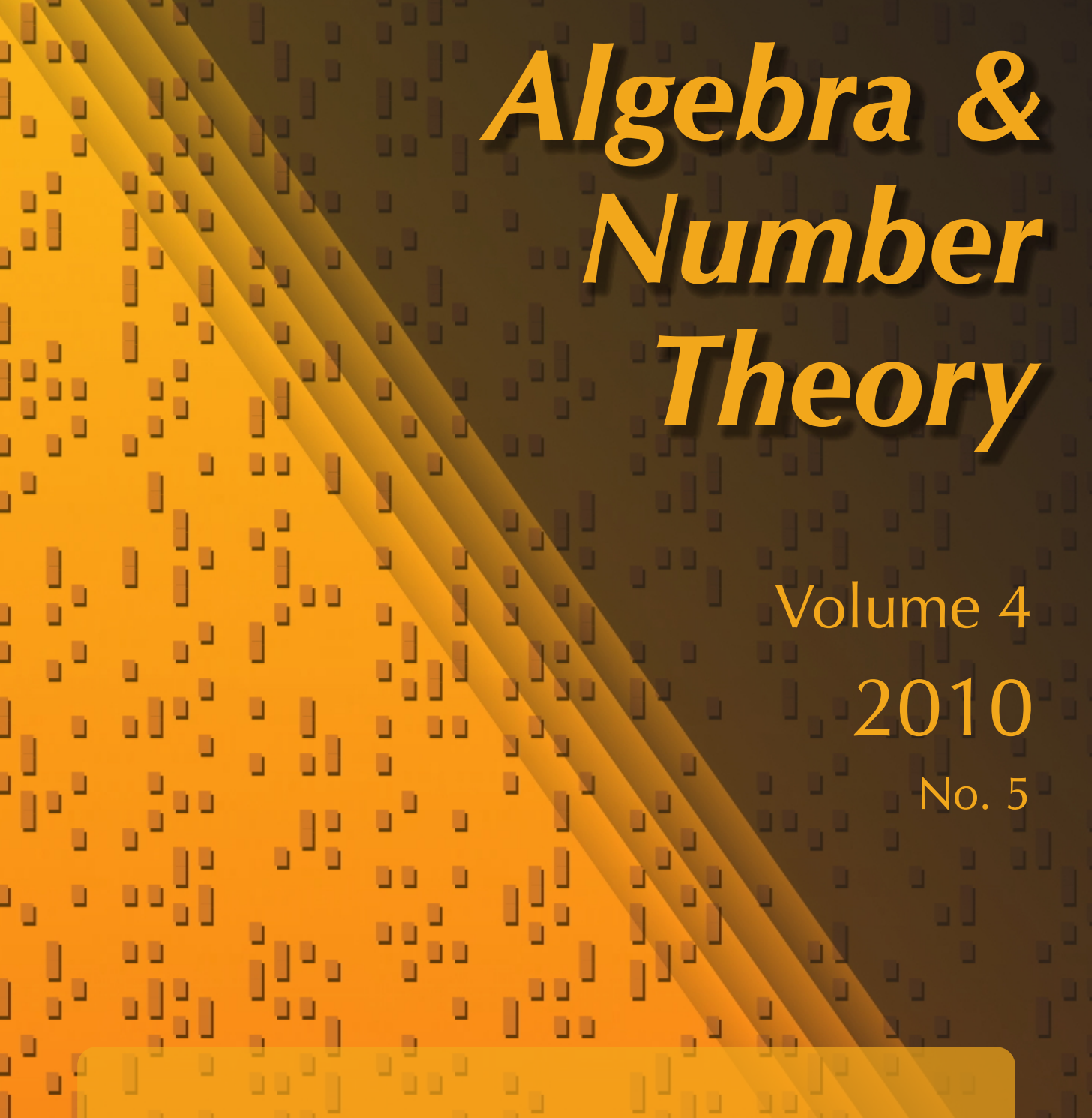

Transverse quiver Grassmannians and bases in affine cluster algebras

U. $\perp\lrcorner\lrcorner-\quad$ Grégoire Dupont

\lrcorner

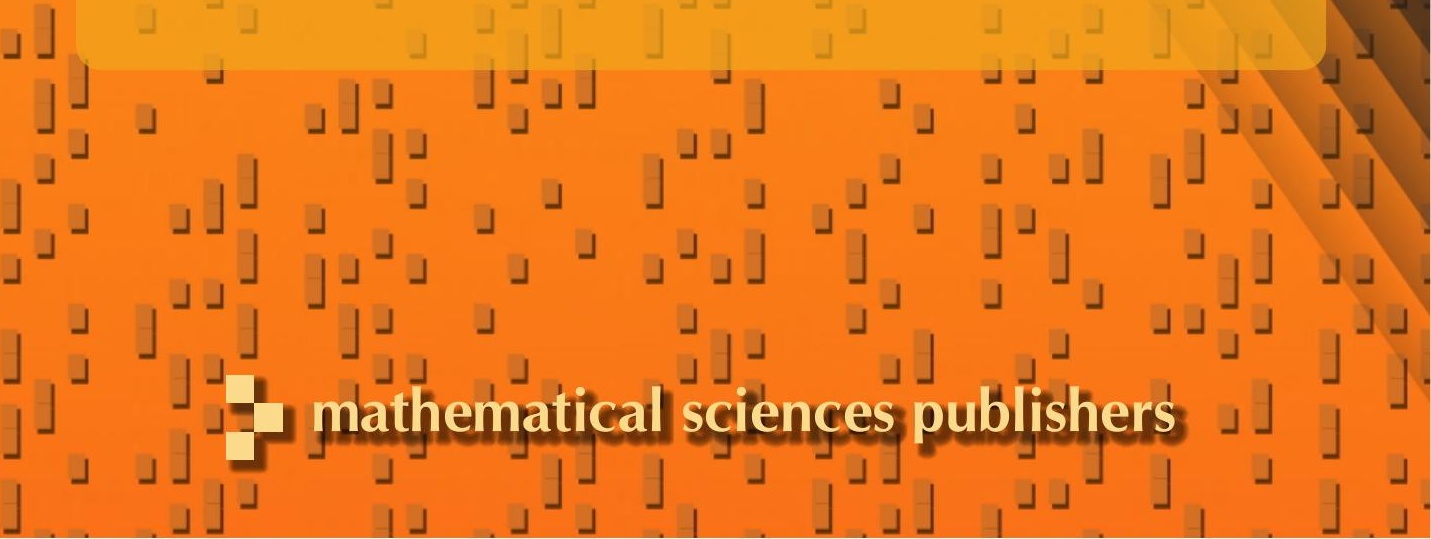




\title{
Transverse quiver Grassmannians and bases in affine cluster algebras
}

\author{
Grégoire Dupont
}

\begin{abstract}
Sherman, Zelevinsky and Cerulli constructed canonically positive bases in cluster algebras associated to affine quivers having at most three vertices. Their constructions involve cluster monomials and normalized Chebyshev polynomials of the first kind evaluated at a certain "imaginary" element in the cluster algebra. Using this combinatorial description, it is possible to define for any affine quiver $Q$ a set $\mathscr{B}(Q)$, which is conjectured to be the canonically positive basis of the acyclic cluster algebra $\mathscr{A}(Q)$.

In this article, we provide a geometric realization of the elements in $\mathscr{B}(Q)$ in terms of the representation theory of $Q$. This is done by introducing an analogue of the Caldero-Chapoton cluster character, where the usual quiver Grassmannian is replaced by a constructible subset called the transverse quiver Grassmannian.
\end{abstract}

1. Introduction $\quad 599$

2. Background, notation and terminology 602

3. Difference properties of higher orders 608

4. Integrable bundles on $\operatorname{rep}_{k}(Q)$ and their characters 615

5. A geometrization of $\mathscr{B}(Q) \quad 617$

6. Examples 620

Acknowledgements $\quad 623$

$\begin{array}{ll}\text { References } & 623\end{array}$

\section{Introduction}

Cluster algebras were introduced by Fomin and Zelevinsky [2002; 2003; 2007; Berenstein et al. 2005] in order to define a combinatorial framework for studying positivity in algebraic groups and canonical bases in quantum groups. Since then, cluster algebras have found applications in various areas of mathematics, such as Lie theory, combinatorics, Teichmüller theory, Poisson geometry and quiver representations.

MSC2000: primary 16G99; secondary 13F99.

Keywords: cluster algebras, canonical bases, Chebyshev polynomials, cluster characters, quiver

Grassmannians. 
A (coefficient-free) cluster algebra $\mathscr{A}$ is a commutative $\mathbb{Z}$-algebra equipped with a distinguished set of generators, called cluster variables, gathered into possibly overlapping sets of fixed cardinality, called clusters. Monomials in variables belonging all to the same cluster are called cluster monomials. According to the Laurent phenomenon [Fomin and Zelevinsky 2002], it is known that $\mathscr{A}$ is a subalgebra of $\mathbb{Z}\left[\boldsymbol{c}^{ \pm 1}\right]$ for any cluster $\boldsymbol{c}$ in $\mathscr{A}$. A nonzero element $y \in \mathscr{A}$ is called positive if $y$ belongs to $\mathbb{Z}_{\geq 0}\left[\boldsymbol{c}^{ \pm 1}\right]$ for any cluster $\boldsymbol{c}$ in $\mathscr{A}$. Following [Cerulli 2009], a $\mathbb{Z}$ basis $\mathscr{B} \subset \mathscr{A}$ is called canonically positive if the semiring of positive elements in $A$ coincides with the set of $\mathbb{Z}_{\geq 0}$-linear combinations of elements of $\mathscr{B}$. Note that if such a basis exists, it is unique.

The problems of existence and description of a canonically positive basis in an arbitrary cluster algebra are still wide open. Both problems were first solved in the particular case of cluster algebras of finite type $\mathbb{A}_{2}$ and affine type $\tilde{A}_{1,1}$ by Sherman and Zelevinsky [2004]. It was later extended by Cerulli [2009] for cluster algebras of affine type $\tilde{A}_{2,1}$. To the best of the author's knowledge, these are the only known constructions of canonically positive bases in cluster algebras.

Using categorifications of acyclic cluster algebras with cluster categories and cluster characters, it is possible to rephrase Sherman-Zelevinsky and Cerulli constructions in order to place them in the more general context of acyclic cluster algebras associated to arbitrary affine quivers.

If $Q$ is an acyclic quiver and $\boldsymbol{u}$ is a $Q_{0}$-tuple of indeterminates over $\mathbb{Z}$, we denote by $\mathscr{A}(Q)$ the acyclic cluster algebra with initial seed $(Q, \boldsymbol{u})$. We denote by $\mathscr{C}_{Q}$ the associated cluster category (over the field $\boldsymbol{k}$ of complex numbers) and by $X_{?}: \mathrm{Ob}\left(\mathscr{C}_{Q}\right) \rightarrow \mathbb{Z}\left[\boldsymbol{u}^{ \pm 1}\right]$ the Caldero-Chapoton map on $\mathscr{C}_{Q}$, also called the (canonical) cluster character (see Section 2 for details). When $Q$ is an affine quiver with positive minimal imaginary root $\delta$, we set

$$
\mathscr{B}(Q)=\mathcal{M}(Q) \sqcup\left\{F_{n}\left(X_{\delta}\right) X_{R} \mid n \geq 1 \text { and } R \text { is a regular rigid } \boldsymbol{k} Q \text {-module }\right\}
$$

where $M(Q)$ denotes the set of cluster monomials in $\mathscr{A}(Q), F_{n}$ denotes the $n$-th normalized Chebyshev polynomial of the first kind and $X_{\delta}$ is the evaluation of $X_{\text {? }}$ ? at any quasisimple module in a homogeneous tube of the Auslander-Reiten quiver $\Gamma(\boldsymbol{k} Q$-mod) of $\boldsymbol{k} Q$-mod.

If $Q$ is of type $\tilde{\mathbb{A}}_{1,1}$ or $\tilde{\mathbb{A}}_{2,1}$, the set $\mathscr{B}(Q)$ coincides with the canonically positive basis constructed in [Sherman and Zelevinsky 2004] and [Cerulli 2009], respectively. It was conjectured in [Dupont 2010, Conjecture 7.10] that, for any affine quiver $Q$, the set $\mathscr{B}(Q)$ is the canonically positive basis of $\mathscr{A}(Q)$. Using the generic basis, it is possible to prove that, for any affine quiver $Q$, the set $\mathscr{B}(Q)$ is a $\mathbb{Z}$-basis in $\mathscr{A}(Q)$ [Dupont 2008; Ding et al. 2009]. Nevertheless, it is not known if this basis is the canonically positive basis in general. 
An essential problem in investigating this question is that the elements of the form $F_{n}\left(X_{\delta}\right) X_{R}$ are defined combinatorially, and as yet have no representationtheoretic or geometric interpretation. The aim of this article is to provide such an interpretation.

Extending the idea of Caldero and Chapoton [2006], for any integrable bundle $\mathscr{F}_{F}$ on $\operatorname{rep}_{k}(Q)$ (see Section 4 for definitions), we define a map $\theta_{\mathscr{F}}$, called the character associated to $\mathscr{F}$, from the set of objects in $\mathscr{C}_{Q}$ to the ring $\mathbb{Z}\left[\boldsymbol{u}^{ \pm 1}\right]$. With this terminology, the Caldero-Chapoton map $X$ ? is the character $\theta_{\mathrm{Gr}}$, where $\mathrm{Gr}: M \mapsto \operatorname{Gr}(M)$ denotes the integrable bundle of quiver Grassmannians.

For any indecomposable $\boldsymbol{k} Q$-module $M$, we introduce a constructible subset $\operatorname{Tr}(M) \subset \operatorname{Gr}(M)$, called the transverse quiver Grassmannian. We prove that the bundle $\operatorname{Tr}: M \mapsto \operatorname{Tr}(M)$ is integrable on $\operatorname{rep}_{\boldsymbol{k}}(Q)$ and that the elements in $\mathscr{B}(Q)$ can be described using the associated character $\theta_{\operatorname{Tr}}$. More precisely, we prove that for any $l \geq 1$,

$$
F_{l}\left(X_{\delta}\right)=\theta_{\mathrm{Tr}}(M),
$$

where $M$ is any indecomposable $\boldsymbol{k} Q$-module with dimension vector $l \delta$. It turns out that $\theta_{\mathrm{Tr}}$, unlike $\theta_{\mathrm{Gr}}$, is independent of the tube containing $M$. In particular, it takes the same values if $M$ belongs to a homogeneous or to an exceptional tube. This is surprising since the usual quiver Grassmannians of two indecomposable modules of dimension $l \delta$ belonging to tubes of different ranks are in general completely different.

Moreover, if $R$ is an indecomposable regular rigid $\boldsymbol{k} Q$-module, then

$$
F_{l}\left(X_{\delta}\right) X_{R}=\theta_{\operatorname{Tr}}(M),
$$

where $M$ is the unique indecomposable $k Q$-module of dimension $l \delta+\operatorname{dim} R$.

As a consequence, we obtain the following description of the set $\mathscr{B}(Q)$ :

$\mathscr{B}(Q)=\left\{\begin{array}{l|l}\theta_{\operatorname{Tr}}(M \oplus R) & \begin{array}{l}M \text { is an indecomposable (or zero) regular } \boldsymbol{k} Q \text {-module, } \\ R \text { is any rigid object in } \mathscr{C}_{Q} \text { such that } \operatorname{Ext}_{\mathscr{C}_{Q}}^{1}(M, R)=0\end{array}\end{array}\right\}$.

This paper is organized as follows. In Section 2, we start by recalling several results concerning Chebyshev and generalized Chebyshev polynomials. Then we recall necessary background on cluster categories and cluster characters associated to acyclic and especially affine quivers. Finally, we recall the known results concerning constructions of bases in affine cluster algebras.

In Section 3, we use the combinatorics of generalized Chebyshev polynomials to prove relations for cluster characters associated to regular $\boldsymbol{k} Q$-modules when $Q$ is an affine quiver with minimal imaginary root $\delta$. These relations are generalizations of the difference property [Dupont 2008], used to compute the difference between cluster characters evaluated at indecomposable modules of dimension vector $\delta$ in different tubes. 
Section 4 introduces the notions of integrable bundles on $\operatorname{rep}_{k}(Q)$ and associated characters for any acyclic quiver. With this terminology, the Caldero-Chapoton map is the character associated to the quiver Grassmannian bundle. For affine quivers, we introduce the integrable bundle $\mathrm{Tr}$ of the Grassmannian of transverse submodules and see that it coincides with the Caldero-Chapoton map on rigid objects in the cluster category.

In Section 5, we prove that the elements in $\mathscr{B}(Q)$ can be expressed as values of the character $\theta_{\operatorname{Tr}}$ associated to the integrable bundle $\operatorname{Tr}$ of $\operatorname{rep}_{\boldsymbol{k}}(Q)$. This provides a geometrization of the set $\mathscr{B}(Q)$.

In Section 6, we illustrate some of our results for quivers of affine types $\tilde{\mathbb{A}}_{1,1}$ and $\tilde{\mathbb{A}}_{2,1}$, putting [Sherman and Zelevinsky 2004; Cerulli 2009] into context.

\section{Background, notation and terminology}

Given a quiver $Q$, we denote by $Q_{0}$ its set of arrows and by $Q_{1}$ its set of vertices. We always assume that $Q_{0}, Q_{1}$ are finite sets and that the underlying unoriented graph of $Q$ is connected. A quiver is called acyclic if it does not contain any oriented cycles.

We now fix an acyclic quiver $Q$ and a $Q_{0}$-tuple $\boldsymbol{u}=\left(u_{i} \mid i \in Q_{0}\right)$ of indeterminates over $\mathbb{Z}$. We denote by $\mathscr{A}(Q)$ the coefficient-free cluster algebra with initial seed $(Q, \boldsymbol{u})$.

Chebyshev polynomials and their generalizations. Chebyshev (respectively generalized Chebyshev) polynomials are orthogonal polynomials in one variable (respectively several variables) playing an important role in the context of cluster algebras associated to representation-infinite quivers [Sherman and Zelevinsky 2004; Caldero and Zelevinsky 2006] (respectively [Dupont 2009; 2010]). We recall some basic results concerning these polynomials.

For any $l \geq 0$, the $l$-th (normalized) Chebyshev polynomial of the first kind is the polynomial $F_{l}$ in $\mathbb{Z}[x]$ defined inductively by

$$
F_{0}(x)=2, \quad F_{1}(x)=x, \quad \text { and } \quad F_{l}(x)=x F_{l-1}(x)-F_{l-2}(x) \quad \text { for any } l \geq 2 .
$$

$F_{l}$ is characterized by the following identity in $\mathbb{Z}\left[t, t^{-1}\right]$ :

$$
F_{l}\left(t+t^{-1}\right)=t^{l}+t^{-l} .
$$

These polynomials first appeared in the context of cluster algebras in [Sherman and Zelevinsky 2004].

For any $l \geq 0$, the $l$-th (normalized) Chebyshev polynomial of the second kind is the polynomial $S_{l}$ in $\mathbb{Z}[x]$ defined inductively by

$$
S_{0}(x)=1, \quad S_{1}(x)=x, \quad \text { and } \quad S_{l}(x)=x S_{l-1}(x)-S_{l-2}(x) \quad \text { for any } l \geq 2 .
$$


$S_{l}$ is characterized by the following identity in $\mathbb{Z}\left[t, t^{-1}\right]$ :

$$
S_{l}\left(t+t^{-1}\right)=\sum_{k=0}^{n} t^{n-2 k} .
$$

Chebyshev polynomials of the Second kind first appeared in the context of cluster algebras in [Caldero and Zelevinsky 2006]. For any $l \geq 1, S_{l}(x)$ is the polynomial given by

$$
S_{l}(x)=\operatorname{det}\left[\begin{array}{ccccc}
x & 1 & & & (0) \\
1 & x & \ddots & & \\
& \ddots & \ddots & \ddots & \\
& & \ddots & \ddots & 1 \\
(0) & & & 1 & x
\end{array}\right],
$$

where the matrix is tridiagonal in $M_{l}(\mathbb{Z}[x])$. The two kinds of Chebyshev polynomials are related by

$$
F_{l}(x)=S_{l}(x)-S_{l-2}(x)
$$

for any $l \geq 1$, with the convention that $S_{-1}(x)=0$.

Fix a family $\left\{x_{i} \mid i \geq 1\right\}$ of indeterminates over $\mathbb{Z}$. For any $l \geq 0$, the $l$-th generalized Chebyshev polynomial is the polynomial in $\mathbb{Z}\left[x_{1}, \ldots, x_{l}\right]$ defined inductively by $P_{0}=1, P_{1}\left(x_{1}\right)=x_{1}$, and

$$
P_{l}\left(x_{1}, \ldots, x_{l}\right)=x_{l} P_{l-1}\left(x_{1}, \ldots, x_{l-1}\right)-P_{l-2}\left(x_{1}, \ldots, x_{l-2}\right) \quad \text { for any } l \geq 2 .
$$

Equivalently,

$$
P_{l}\left(x_{1}, \ldots, x_{l}\right)=\operatorname{det}\left[\begin{array}{rrrrr}
x_{l} & 1 & & & (0) \\
1 & x_{l-1} & \ddots & & \\
& \ddots & \ddots & \ddots & \\
& & \ddots & \ddots & 1 \\
(0) & & & 1 & x_{1}
\end{array}\right] \text {, }
$$

where the matrix is tridiagonal in $M_{l}\left(\mathbb{Z}\left[x_{1}, \ldots, x_{l}\right]\right)$. These polynomials first appeared in the context of cluster algebras in [Dupont 2009] under the name of generalized Chebyshev polynomials of infinite rank, and similar polynomials also arose in the context of cluster algebras in [Yang and Zelevinsky 2008; Dupont 2010].

Cluster categories and cluster characters. Let $\boldsymbol{k} Q$-mod be the category of finitely generated left-modules over the path algebra $k Q$ of $Q$. As usual, this category will be identified with the category $\operatorname{rep}_{k}(Q)$ of finite-dimensional representations of $Q$ over $\boldsymbol{k}$.

For any vertex $i \in Q_{0}$, we denote by $S_{i}$ the simple module associated to $i$, by $P_{i}$ its projective cover, and by $I_{i}$ its injective hull. We denote by $\langle-,-\rangle$ the 
homological Euler form defined on $\boldsymbol{k} Q$-mod by

$$
\langle M, N\rangle=\operatorname{dim} \operatorname{Hom}_{k}(M, N)-\operatorname{dim} \operatorname{Ext}_{k Q}^{1}(M, N)
$$

for any two $\boldsymbol{k} Q$-modules $M, N$. Since $Q$ is acyclic, $\boldsymbol{k} Q$ is a finite-dimensional hereditary algebra, and therefore $\langle-,-\rangle$ is well-defined on the Grothendieck group $K_{0}(\boldsymbol{k} Q$-mod).

For any $\boldsymbol{k} Q$-module $M$, the dimension vector of $M$ is

$$
\operatorname{dim} M=\left(\operatorname{dim} \operatorname{Hom}_{k}\left(P_{i}, M\right)\right)_{i \in Q_{0}} \in \mathbb{N}^{Q_{0}} .
$$

Viewed as a representation of $Q, \operatorname{dim} M=(\operatorname{dim} M(i))_{i \in Q_{0}}$ where $M(i)$ is the $\boldsymbol{k}$ vector space at vertex $i$ in the representation $M$ of $Q$. The dimension vector map dim induces an isomorphism of abelian groups

$$
\operatorname{dim}: K_{0}(\boldsymbol{k} Q-\bmod ) \stackrel{\sim}{\rightarrow} \mathbb{Z}^{Q_{0}}
$$

sending the class of the simple $S_{i}$ to the $i$-th vector of the canonical basis of $\mathbb{Z}^{Q_{0}}$.

The cluster category was introduced in [Buan et al. 2006] (see also [Caldero et al. 2006] for Dynkin type $A$ ) in order to define a categorical framework for studying the cluster algebra $\mathscr{A}(Q)$. Let $D^{b}(\boldsymbol{k} Q$-mod) be the bounded derived category of $\boldsymbol{k} Q$-mod with shift functor [1] and Auslander-Reiten translation $\tau$. The cluster category is the orbit category $\mathscr{C}_{Q}$ of the auto-functor $\tau^{-1}[1]$ in $D^{b}(\boldsymbol{k} Q$-mod). It is a 2-Calabi-Yau triangulated category. The set of isoclasses of indecomposable objects in $\mathscr{C}_{Q}$ can be identified with the union of the set of isoclasses of indecomposable $\boldsymbol{k} Q$-modules and the set of isoclasses of shifts of indecomposable projective $\boldsymbol{k} Q$-modules [Keller 2005; Buan et al. 2006]. In particular, every object $M$ in $\mathscr{C}_{Q}$ can be uniquely (up to isomorphism) decomposed into

$$
M=M_{0} \oplus P_{M}[1],
$$

where $M_{0}$ is a $\boldsymbol{k} Q$-module and $P_{M}$ is a projective $\boldsymbol{k} Q$-module.

Given a representation $M$ of $Q$, the quiver Grassmannian of $M$ is the $\operatorname{set} \operatorname{Gr}(M)$ of all subrepresentations of $M$. For any element $\boldsymbol{e} \in \mathbb{Z}^{Q_{0}}$, the set

$$
\operatorname{Gr}_{\boldsymbol{e}}(M)=\{N \text { submodule of } M \mid \operatorname{dim} N=\boldsymbol{e}\}
$$

is a projective variety. We denote by $\chi\left(\mathrm{Gr}_{e}(M)\right)$ its Euler characteristic with respect to the singular cohomology with rational coefficients.

Definition 2.1 [Caldero and Chapoton 2006]. The Caldero-Chapoton map is the map

$$
X_{?}: \mathrm{Ob}\left(\mathscr{C}_{Q}\right) \rightarrow \mathbb{Z}\left[\boldsymbol{u}^{ \pm 1}\right]
$$

defined by: 
- For any $i \in Q_{0}$,

$$
X_{P_{i}[1]}=u_{i}
$$

- If $M$ is an indecomposable $k Q$-module, then

$$
X_{M}=\sum_{\boldsymbol{e} \in \mathbb{N} Q_{0}} \chi\left(\operatorname{Gr}_{\boldsymbol{e}}(M)\right) \prod_{i \in Q_{0}} u_{i}^{-\left\langle\boldsymbol{e}, S_{i}\right\rangle-\left\langle S_{i}, \operatorname{dim} M-\boldsymbol{e}\right\rangle} .
$$

- For any two objects $M, N$ in $\mathscr{C}_{Q}$,

$$
X_{M \oplus N}=X_{M} X_{N} .
$$

Note that (2-1) also holds for decomposable modules.

Caldero and Keller [2006, Theorem 4] proved that $X$ ? induces a 1-1 correspondence between the set of isoclasses of indecomposable rigid objects (that is, without self-extensions) in $\mathscr{C}_{Q}$ and the set of cluster variables in $\mathscr{A}(Q)$. Moreover, $X_{\text {? }}$ induces a 1-1 correspondence between the set of isoclasses of cluster-tilting objects in $\mathscr{C}_{Q}$ and the set of clusters in $\mathscr{A}(Q)$. In particular, we have the following description of cluster monomials in $\mathscr{A}(Q)$ :

$$
\mathcal{M}(Q)=\left\{X_{M} \mid M \text { is rigid in } \mathscr{C}_{Q}\right\} .
$$

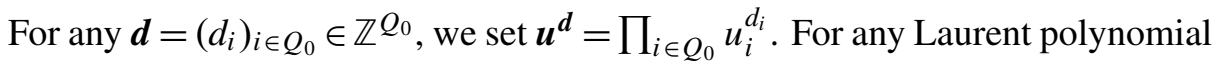
$L \in \mathbb{Z}\left[\boldsymbol{u}^{ \pm 1}\right]$, the denominator vector of $L$ is the $Q_{0}$-tuple $\operatorname{den}(L) \in \mathbb{Z}^{Q_{0}}$ such that there exists a polynomial $P\left(u_{i} \mid i \in Q_{0}\right)$ not divisible by any $u_{i}$ such that

$$
L=\frac{P\left(u_{i} \mid i \in Q_{0}\right)}{\boldsymbol{u}^{\operatorname{den}(L)}} .
$$

We define the dimension vector map $\operatorname{dim}_{\mathscr{C}_{Q}}$ on $\mathscr{C}_{Q}$ by setting $\operatorname{dim}_{\mathscr{C}_{Q}} M=\operatorname{dim} M$ if $M$ is a $\boldsymbol{k} Q$-module and $\operatorname{dim}_{\mathscr{C}_{Q}} P_{i}[1]=-\operatorname{dim} S_{i}$ and extending by additivity. Note that, for any $k Q$-module $M$, we have $\operatorname{dim} M=\operatorname{dim}_{\mathscr{C}_{Q}}(M)$; we will abuse notation and write $\operatorname{dim} M$ for any object in $\mathscr{C}_{Q}$. Caldero and Keller's denominator theorem [2006, Theorem 3] relates the denominator vector of the character with the dimension vector of the corresponding object in the cluster category:

$$
\operatorname{den}\left(X_{M}\right)=\operatorname{dim} M,
$$

for any object $M$ in $\mathscr{b}_{Q}$.

Representation theory of affine quivers. We shall briefly recall some well-known facts concerning the representation theory of affine quivers. We refer the reader to [Simson and Skowroński 2007; Ringel 1984] for details.

We now fix an affine quiver $Q$, that is, an acyclic quiver of type $\tilde{\mathbb{A}}_{n},(n \geq 1)$, $\tilde{\mathbb{D}}_{n},(n \geq 4), \tilde{\mathbb{E}}_{n},(n=6,7,8)$. We will say that a quiver is of affine type $\tilde{\mathbb{A}}_{r, s}$ if it is an orientation of an affine diagram of affine type $\tilde{\mathbb{A}}_{r+s-1}$, with $r$ arrows going 
clockwise and $s$ arrows going counterclockwise. Let $\mathfrak{g}_{Q}$ denote the Kac-Moody algebra associated to $Q$.

We denote by $\Phi_{>0}$ the set of positive roots of $\mathfrak{g}_{Q}$, by $\Phi_{>0}^{\text {re }}$ the set of positive real roots and by $\Phi_{>0}^{\mathrm{im}}$ the set of positive imaginary roots. Since $Q$ is affine, there exists a unique $\delta \in \Phi_{>0}$ such that $\Phi_{>0}^{\mathrm{im}}=\mathbb{Z}_{>0} \delta$. We always identify the root lattice of $\mathfrak{g}_{Q}$ with $\mathbb{Z}^{Q_{0}}$ by sending the $i$-th simple root of $\mathfrak{g}_{Q}$ to the $i$-th vector of the canonical basis of $\mathbb{Z}^{Q_{0}}$.

According to Kac's theorem, for any $\boldsymbol{d} \in \mathbb{N}^{Q_{0}}$, there exists an indecomposable representation $M$ such that $\operatorname{dim} M=\boldsymbol{d}$ if and only if $\boldsymbol{d} \in \Phi_{>0}$. Moreover, this representation is unique up to isomorphism if and only if $\boldsymbol{d} \in \Phi_{>0}^{\text {re }}$. A positive root $\boldsymbol{d}$ is called a Schur root if there exists a (necessarily indecomposable) representation $M$ of $Q$ such that $\operatorname{dim} M=\boldsymbol{d}$ and $\operatorname{End}_{\boldsymbol{k}}(M) \simeq \boldsymbol{k}$.

We define a partial order $\leq$ on the root lattice by setting

$$
\boldsymbol{e} \leq \boldsymbol{f} \quad \Longleftrightarrow \quad e_{i} \leq d_{i} \text { for any } i \in Q_{0},
$$

and we set

$$
\boldsymbol{e} \supsetneqq \boldsymbol{f} \quad \text { if } \boldsymbol{e} \leq \boldsymbol{f} \text { and } \boldsymbol{e} \neq \boldsymbol{f} .
$$

The Auslander-Reiten quiver $\Gamma(\boldsymbol{k} Q$-mod $)$ of $\boldsymbol{k} Q$-mod contains infinitely many connected components. There exists a connected component containing all the projective (resp. injective) modules, called the preprojective (resp. preinjective) component of $\Gamma(\boldsymbol{k} Q$-mod) and denoted by $\mathscr{P}$ (resp. $\mathscr{I})$. The other components are called regular. A $\boldsymbol{k} Q$-module $M$ is called preprojective, preinjective, or regular if each indecomposable direct summand of $M$ belongs to a component with the same property.

It is convenient to introduce the so-called defect form on $\mathbb{Z}^{Q_{0}}$. It is given by

$$
\partial_{?}: \mathbb{Z}^{Q_{0}} \rightarrow \mathbb{Z}, \quad \boldsymbol{e} \mapsto \partial_{\boldsymbol{e}}=\langle\delta, \boldsymbol{e}\rangle .
$$

By definition, the defect $\partial_{M}$ of a $\boldsymbol{k} Q$-module $M$ is the defect $\partial_{\operatorname{dim}} M$ of its dimension vector. It is well-known that an indecomposable $\boldsymbol{k} Q$-module $M$ is preprojective, preinjective, or regular depending on whether $\partial_{M}$ is negative, positive or zero.

The regular components in $\Gamma(\boldsymbol{k} Q$-mod $)$ form a $\mathbb{P}^{1}(\boldsymbol{k})$-family of tubes. Thus, for every tube $\mathcal{T}$, there exists an integer $p \geq 1$, called the rank of $\mathcal{T}$, such that $\mathscr{T} \simeq \mathbb{Z} \mathbb{A}_{\infty} /\left(\tau^{p}\right)$. The tubes of rank 1 are called homogeneous, while those of rank $p>1$ are called exceptional. At most three tubes are exceptional in $\Gamma(\boldsymbol{k} Q$-mod $)$. It is well-known that the full subcategory of $k Q$-mod formed by the objects in any tube $\mathscr{T}$ is standard, that is, isomorphic to the mesh category of $\mathscr{T}$. It is also known that there are neither morphisms nor extensions between pairwise distinct tubes.

An indecomposable regular $\boldsymbol{k} Q$-module $M$ is called quasisimple if it is the mouth of the tube, or equivalently, if it does not contain any proper regular submodule. For any quasisimple module $R$ in a tube $\mathcal{T}$ and any integer $l \geq 1$, we denote by 
$R^{(l)}$ the unique indecomposable $k Q$-module with quasisocle $R$ and quasilength $l$. For any indecomposable regular $\boldsymbol{k} Q$-module $R^{(l)}$, we denote by

$$
\mathrm{qsoc} R^{(l)}=R
$$

the quasisocle of $M$ and by

$$
\operatorname{qrad} R^{(l)}=R^{(l-1)}
$$

the quasiradical of $M$ with the convention that $R^{(0)}=0$.

For any indecomposable regular $\boldsymbol{k} Q$-module $M$, we have

$$
M \text { is rigid } \Longleftrightarrow \operatorname{dim} M \supsetneqq \delta ; \quad \operatorname{End}_{k Q}(M) \simeq k \Longleftrightarrow \operatorname{dim} M \leq \delta .
$$

Cluster characters associated to modules in tubes are known to be governed by the combinatorics of generalized Chebyshev polynomials. More precisely, it is proved in [Dupont 2009, Theorem 5.1] that for any quasisimple module $M$ in a tube $\mathscr{T}$, we have

$$
X_{M^{(l)}}=P_{l}\left(X_{M}, X_{\tau^{-1} M}, \ldots, X_{\tau^{-l+1} M}\right) .
$$

In particular, if $\mathscr{T}$ is homogeneous, we get $X_{M^{(l)}}=S_{l}\left(X_{M}\right)$, recovering a result of [Caldero and Zelevinsky 2006].

In [Dupont 2009, Theorem 7.2], generalized Chebyshev polynomials provide multiplication formulas for cluster characters associated to indecomposable regular $\boldsymbol{k} Q$-modules. The following theorem will be essential in the proofs.

Theorem 2.2 [Dupont 2009]. Let $Q$ be an affine quiver and $\mathcal{T}$ be a tube of rank $p$ in $\Gamma\left(\boldsymbol{k} Q\right.$-mod). Let $R_{i}, i \in \mathbb{Z}$ denote the quasisimple modules in $\mathcal{T}$ ordered such that $\tau R_{i} \simeq R_{i-1}$ and $R_{i+p} \simeq R_{i}$ for any $i \in \mathbb{Z}$. Let $m, n>0$ be integers and $j \in[0, p-1]$. Then, for every $k \in \mathbb{Z}$ such that $0<j+k p \leq n$ and $m \geq n-j-k p$, we have the identity

$$
X_{R_{j}^{(m)}} X_{R_{0}^{(n)}}=X_{R_{0}^{(m+j+k p)}} X_{R_{j}^{(n-j-k p)}}+X_{R_{0}^{(j+k p-1)}} X_{R_{n+1}^{(m+j+k p-n-1)}} .
$$

Bases in affine cluster algebras. We shall now review some results concerning the construction of $\mathbb{Z}$-bases in cluster algebras associated to affine quivers. In this section, $Q$ still denotes an affine quiver with positive minimal imaginary root $\delta$.

If $M, N$ are quasisimple modules in distinct homogeneous tubes, then $X_{M}=X_{N}$; see [Dupont 2008], for example. We denote this common value by $X_{\delta}$, and call it the generic variable of dimension $\delta$, as in that earlier paper.

Theorem 2.3 [Dupont 2008; Ding et al. 2009]. Let $Q$ be an affine quiver. Then

$$
G(Q)=\mathcal{M}(Q) \sqcup\left\{X_{\delta}^{l} X_{R} \mid l \geq 1, R \text { is a regular rigid } \boldsymbol{k} Q \text {-module }\right\}
$$

is a $\mathbb{Z}$-basis of $\mathscr{A}(Q)$.

Moreover, den induces a 1-1 correspondence from $\mathscr{G}(Q)$ to $\mathbb{Z}^{Q_{0}}$. 
The set $\mathscr{G}(Q)$ is called the generic basis of $\mathscr{A}(Q)$.

Since $F_{l}$ and $S_{l}$ are monic polynomials of degree $l$, it follows that, for any affine quiver $Q$, the sets

$$
\mathscr{B}(Q)=\mathcal{M}(Q) \sqcup\left\{F_{l}\left(X_{\delta}\right) X_{R} \mid l \geq 1, R \text { is a regular rigid } \boldsymbol{k} Q \text {-module }\right\}
$$

and

$$
\mathscr{C}(Q)=\mathcal{M}(Q) \sqcup\left\{S_{l}\left(X_{\delta}\right) X_{R} \mid l \geq 1, R \text { is a regular rigid } \boldsymbol{k} Q \text {-module }\right\}
$$

are $\mathbb{Z}$-bases of the cluster algebra $\mathscr{A}(Q)$.

When $Q$ is the Kronecker quiver, $\mathscr{B}(Q)$ coincides with the canonically positive basis constructed in [Sherman and Zelevinsky 2004] and $\mathscr{C}(Q)$ coincides with the basis constructed in [Caldero and Zelevinsky 2006]. When $Q$ is a quiver of affine type $\tilde{\mathbb{A}}_{2,1}$, the basis $\mathscr{B}(Q)$ is the canonically positive basis of $\mathscr{A}(Q)$ constructed in [Cerulli 2009].

Since $X_{\delta}=X_{M}$ for any quasisimple module $M$ in a homogeneous tube, it follows that $S_{l}\left(X_{\delta}\right)=X_{M^{(l)}}$ for any $l \geq 0$, so the set $\mathscr{C}(Q)$ has an interpretation in terms of the cluster character $X_{\text {? }}$. No such interpretation had been known for the set $\mathscr{B}(Q)$; this paper provides one.

The map $\phi: \mathscr{G}(Q) \rightarrow \mathscr{B}(Q)$ preserving cluster monomials and sending $X_{\delta}^{l} X_{R}$ to $F_{l}\left(X_{\delta}\right) X_{R}$ for any $l \geq 1$ and any rigid regular module $R$ is a 1-1 correspondence. We denote by

$$
\mathfrak{b}_{?}: \mathbb{Z}^{Q_{0}} \stackrel{1: 1}{\longrightarrow} \mathscr{B}(Q), \quad \boldsymbol{d} \mapsto \mathfrak{b}_{\boldsymbol{d}}
$$

the $1-1$ correspondence obtained by composing the bijection above with the one provided in Theorem 2.3.

\section{Difference properties of higher orders}

Here $Q$ still denotes an affine quiver with positive minimal imaginary root $\delta$.

The difference property. In [Dupont 2008] we introduced the difference property, which relates the possibly different values of cluster characters evaluated at different indecomposable representations of dimension $\delta$. The difference property was crucial in that article. It is also an essential ingredient in this one, since transverse Grassmannians will arise precisely from difference properties of higher orders.

This difference property was established in [Dupont 2008] for affine type $\tilde{\mathbb{A}}$ and in [Ding et al. 2009] in general. It can be expressed as follows:

Theorem 3.1 [Dupont 2008; Ding et al. 2009]. If $Q$ is an affine quiver and $M$ is any indecomposable module of dimension $\delta$, then

$$
\mathfrak{b}_{\delta}=X_{\delta}=X_{M}-X_{\mathrm{qrad} M} / \mathrm{qsoc} M,
$$

with the convention that $X_{\mathrm{qrad} M / \mathrm{qsoc} M}=0$ if $M$ is quasisimple. 
Higher-difference properties. The aim of this section is to provide an analogue of Theorem 3.1 for $\mathfrak{b}_{\boldsymbol{d}}$ when $\boldsymbol{d}$ is any positive root with zero defect. We will first consider the imaginary roots and then the real roots of defect zero.

We fix a tube $\mathscr{T}$ in $\Gamma(k Q$-mod) of rank $p \geq 1$. The quasisimples of $\mathscr{T}$ are denoted by $R_{i}$, with $i \in \mathbb{Z} / p \mathbb{Z}$, ordered so that $\tau R_{i} \simeq R_{i-1}$ for any $i \in \mathbb{Z} / p \mathbb{Z}$. Note that for any $l \geq 1$ and $0 \leq k \leq p-1$ and any $i \in \mathbb{Z} / p \mathbb{Z}$, we have $\operatorname{dim} R_{i}^{(l p)}=l \delta \in \Phi_{>0}^{\mathrm{im}}$ and $\operatorname{dim} R_{i}^{(l p+k)} \in \Phi_{>0}^{\text {re }}$ if $k \neq 0$.

The following technical lemma will be used in the proof of Proposition 3.3:

Lemma 3.2. With the notation above, for any $l \geq 1$,

$$
X_{R_{0}^{(l p-1)}} X_{R_{1}^{(p-1)}}=X_{R_{0}^{(p-1)}} X_{R_{1}^{(l p-1)}} .
$$

Proof. We first notice that generalized Chebyshev polynomials are symmetric in the sense that for every $i \in \mathbb{Z}$ and $n \geq 1$,

$$
P_{n}\left(x_{i}, \ldots, x_{i+n-1}\right)=P_{n}\left(x_{i+n-1}, \ldots, x_{i}\right) .
$$

If $l=1$, the result is obvious. We thus fix some $l \geq 2$. For technical convenience, we denote by $R_{i}, i \in \mathbb{Z}$ the quasisimple modules in $\mathscr{T}$ and we assume that $R_{i} \simeq R_{i+p}$ for every $i \in \mathbb{Z}$. Consider the morphism of $\mathbb{Z}$-algebras

$$
\begin{aligned}
\phi: \mathbb{Z}\left[X_{R_{0}}, \ldots, X_{R_{l p-1}}\right] & \rightarrow \mathbb{Z}\left[X_{R_{0}}, \ldots, X_{R_{l p-1}}\right] \\
X_{R_{i}} & \mapsto X_{R_{l p-1-i}} \quad \text { for all } i=0, \ldots, l p-1 .
\end{aligned}
$$

It is well-defined since $X_{R_{0}}, \ldots, X_{R_{p-1}}$ are known to be algebraically independent over $\mathbb{Z}$ (see for example [Dupont 2009]).

According to Theorem 2.2, we have

$$
X_{R_{1}^{(p-1)}} X_{R_{0}^{(l p-1)}}=X_{R_{0}^{(p)}} X_{R_{1}^{(l p-2)}}-X_{R_{p+1}^{((l-1) p-2)}} .
$$

According to [Dupont 2009, Theorem 5.1], each of the $X_{R_{j}}^{(k)}$ appearing above lies in $\mathbb{Z}\left[X_{R_{0}}, \ldots, X_{R_{l p-1}}\right]$. We can thus apply $\phi$ and we get

$$
\phi\left(X_{R_{1}^{(p-1)}}\right) \phi\left(X_{R_{0}^{(l p-1)}}\right)=\phi\left(X_{R_{0}^{(p)}}\right) \phi\left(X_{R_{1}^{(l p-2)}}\right)-\phi\left(X_{R_{p+1}^{((l-1) p-2)}}\right) .
$$

We now compute these images under $\phi$.

$$
\begin{aligned}
\phi\left(X_{R_{1}^{(p-1)}}\right) & =\phi\left(P_{p-1}\left(X_{R_{1}}, \ldots, X_{R_{p-1}}\right)\right) \\
& =P_{p-1}\left(\phi\left(X_{R_{1}}\right), \ldots, \phi\left(X_{R_{p-1}}\right)\right)=P_{p-1}\left(X_{R_{l p-2}}, \ldots, X_{R_{(l-1) p}}\right) \\
& =P_{p-1}\left(X_{R_{(l-1) p}}, \ldots, X_{R_{l p-2}}\right)=X_{R_{(l-1) p}^{(p-1)}}=X_{R_{0}^{(p-1)}}
\end{aligned}
$$




$$
\begin{aligned}
& \phi\left(X_{R_{0}^{(l p-1)}}\right)=\phi\left(P_{l p-1}\left(X_{R_{0}}, \ldots, X_{R_{l p-2}}\right)\right) \\
& =P_{l p-1}\left(\phi\left(X_{R_{0}}\right), \ldots, \phi\left(X_{R_{l p-2}}\right)\right)=P_{l p-1}\left(X_{R_{l p-1}}, \ldots, X_{R_{1}}\right) \\
& =P_{l p-1}\left(X_{R_{1}}, \ldots, X_{R_{l p-1}}\right)=X_{R_{1}^{(l p-1)}} \\
& \phi\left(X_{R_{0}^{(p)}}\right)=\phi\left(P_{p}\left(X_{R_{0}}, \ldots, X_{R_{p-1}}\right)\right)=P_{p}\left(\phi\left(X_{R_{0}}\right), \ldots, \phi\left(X_{R_{p-1}}\right)\right) \\
& =P_{p}\left(X_{R_{l p-1}}, \ldots, X_{R_{(l-1) p}}\right)=P_{p}\left(X_{R_{(l-1) p}}, \ldots, X_{R_{l p-1}}\right) \\
& =X_{R_{(l-1) p}^{(p)}}=X_{R_{0}^{(p)}} \\
& \phi\left(X_{R_{1}^{(l p-2)}}\right)=\phi\left(P_{l p-2}\left(X_{R_{1}}, \ldots, X_{R_{l p-2}}\right)\right) \\
& =P_{l p-2}\left(\phi\left(X_{R_{1}}\right), \ldots, \phi\left(X_{R_{l p-2}}\right)\right)=P_{l p-2}\left(X_{R_{l p-2}}, \ldots, X_{R_{1}}\right) \\
& =P_{l p-2}\left(X_{R_{1}}, \ldots, X_{R_{l p-2}}\right)=X_{R_{1}^{(l p-2)}} \\
& \phi\left(X_{R_{p+1}^{((l-1) p-2)}}\right)=\phi\left(P_{(l-1) p-2}\left(X_{R_{p+1}}, \ldots, X_{R_{l p-2}}\right)\right) \\
& =P_{(l-1) p-2}\left(\phi\left(X_{R_{p+1}}\right), \ldots, \phi\left(X_{R_{l p-2}}\right)\right) \\
& =P_{(l-1) p-2}\left(X_{R_{(l-1) p-2}}, \ldots, X_{R_{1}}\right) \\
& =P_{(l-1) p-2}\left(X_{R_{1}}, \ldots, X_{R_{(l-1) p-2}}\right)=X_{R_{1}^{((l-1) p-2)}} .
\end{aligned}
$$

Substituting in (3-1), we get

$$
\begin{aligned}
X_{R_{0}^{(p-1)}} X_{R_{1}^{(l p-1)}} & =X_{R_{0}^{(p)}} X_{R_{1}^{(l p-2)}}-X_{R_{1}^{(l-1) p-2)}} \\
& =X_{R_{0}^{(p)}} X_{R_{1}^{(l p-2)}}-X_{R_{p+1}^{(l-1) p-2)}}=X_{R_{1}^{(p-1)}} X_{R_{0}^{(l p-1)} .}
\end{aligned}
$$

We can now prove some higher-difference properties for imaginary roots.

Proposition 3.3. Fix $l \geq 1$. Then for any indecomposable representation $M$ in $\operatorname{rep}_{k}(Q, l \delta)$, we have

$$
\mathfrak{b}_{l \delta}=F_{l}\left(X_{\delta}\right)=X_{M}-X_{\mathrm{qrad}} M / \operatorname{qsoc} M,
$$

with the convention that $X_{\mathrm{qrad}} M / \mathrm{qsoc} M=0$ if $M$ is quasisimple.

Proof. We first treat the case where $M$ is an indecomposable representation of dimension $l \delta$ in a homogeneous tube. It is not necessary to prove it separately but in this particular case, the proof is straightforward. We write $M=R^{(l)}$ for some quasisimple module $R$ in a homogeneous tube. If $l=1$, the proposition follows from Theorem 3.1. If $l \geq 2$, qrad $M / \operatorname{qsoc} M \simeq R^{(l-2)}$, so that

$$
\begin{aligned}
X_{M}-X_{\mathrm{qrad} M / \operatorname{qsoc} M} & =X_{R^{(l)}}-X_{R^{(l-2)}} \\
& =S_{l}\left(X_{R}\right)-S_{l-2}\left(X_{R}\right)=F_{l}\left(X_{R}\right)=F_{l}\left(X_{\delta}\right) .
\end{aligned}
$$


We now assume that $M$ is an indecomposable representation of dimension $l \delta$ in an exceptional tube $\mathcal{T}$ of rank $p \geq 2$. We denote by $R_{0}, \ldots, R_{p-1}$ the quasisimples in $\mathscr{T}$ ordered such that $\tau R_{i} \simeq R_{i-1}$ for any $i \in \mathbb{Z} / p \mathbb{Z}$. We can thus write $M \simeq R_{i}^{(l p)}$ for some $i \in \mathbb{Z} / p \mathbb{Z}$. Without loss of generality, we assume that $i=0$. In order to simplify the notation, for any $l \geq 1$, we write

$$
\Delta_{l}=X_{R_{0}^{(l p)}}-X_{R_{1}^{(l p-2)}} .
$$

We thus have to prove that for any $l \geq 1$,

$$
\Delta_{l}=F_{l}\left(X_{\delta}\right) .
$$

The central tool in this proof is Theorem 2.2. According to Theorem 3.1, we have

$$
X_{\delta}=X_{R_{0}^{(p)}}-X_{R_{1}^{(p-2)}}
$$

so the proposition holds for $l=1$.

We now prove it for $l=2$. We have

$$
\begin{aligned}
F_{2}\left(X_{\delta}\right) & =X_{\delta}^{2}-2=\left(X_{R_{0}^{(p)}}-X_{R_{1}^{(p-2)}}\right)^{2}-2 \\
& =X_{R_{0}^{(p)}}^{2}+X_{R_{1}^{(p-2)}}^{2}-2 X_{R_{0}^{(p)}} X_{R_{1}^{(p-2)}}-2,
\end{aligned}
$$

but according to the almost split multiplication formula [Caldero and Chapoton 2006, Proposition 3.10], we have

$$
X_{R_{0}^{(p-1)}} X_{R_{1}^{(p-1)}}=X_{R_{0}^{(p)}} X_{R_{1}^{(p-2)}},
$$

so

$$
F_{2}\left(X_{\delta}\right)=X_{R_{0}^{(p)}}^{2}-2 X_{R_{0}^{(p-1)}} X_{R_{1}^{(p-1)}}+X_{R_{1}^{(p-2)}}^{2} .
$$

But, according to Theorem 2.2, we have

$$
X_{R_{0}^{(p)}}^{2}=X_{R_{0}^{(p)}} X_{R_{0}^{(p)}}=X_{R_{0}^{(2 p)}}+X_{R_{0}^{(p-1)}} X_{R_{1}^{(p-1)}},
$$

so that finally

$$
F_{2}\left(X_{\delta}\right)=X_{R_{0}^{(2 p)}}-X_{R_{0}^{(p-1)}} X_{R_{1}^{(p-1)}}+X_{R_{1}^{(p-2)}}^{2}
$$

Thus,

$$
\begin{aligned}
F_{2}\left(X_{\delta}\right)=\Delta_{2} & \Longleftrightarrow-X_{R_{1}^{(2 p-2)}}=X_{R_{1}^{(p-2)}}^{2}-X_{R_{0}^{(p-1)}} X_{R_{1}^{(p-1)}} \\
& \Longleftrightarrow X_{R_{1}^{(2 p-2)}}+X_{R_{1}^{(p-2)}}^{2}-X_{R_{0}^{(p-1)}} X_{R_{1}^{(p-1)}}=0 .
\end{aligned}
$$

But

$$
X_{R_{1}^{(2 p-2)}}=-X_{R_{1}^{(p-3)}} X_{R_{0}^{(p-1)}}+X_{R_{1}^{(p-2)}} X_{R_{p-1}^{(p)}}
$$


So

$$
\begin{aligned}
F_{2}\left(X_{\delta}\right)=\Delta_{2} & \Longleftrightarrow X_{R_{1}^{(p-2)}}^{2}+X_{R_{1}^{(p-2)}} X_{R_{p-1}^{(p)}}-X_{R_{0}^{(p-1)}} X_{R_{1}^{(p-1)}}-X_{R_{0}^{(p-1)}} X_{R_{1}^{(p-3)}}=0 \\
& \Longleftrightarrow X_{R_{1}^{(p-2)}}\left[X_{R_{1}^{(p-2)}}+X_{R_{p-1}^{(p)}}\right]-X_{R_{0}^{(p-1)}}\left[X_{R_{1}^{(p-3)}}+X_{R_{1}^{(p-1)}}\right]=0 .
\end{aligned}
$$

Theorem 2.2 gives

$$
X_{R_{p-1}} X_{R_{0}^{(p-1)}}=X_{R_{1}^{(p-2)}}+X_{R_{p-1}^{(p)}} \quad \text { and } \quad X_{R_{1}^{(p-2)}} X_{R_{p-1}}=X_{R_{1}^{(p-3)}}+X_{R_{1}^{(p-1)}},
$$

so

$$
F_{2}\left(X_{\delta}\right)=\Delta_{2}
$$

and the proposition is proved for $l=2$.

For $l>2$, we will use the three-term relations for first kind Chebyshev polynomials

$$
F_{l}(x)=x F_{l-1}(x)-F_{l-2}(x) .
$$

Thus, it is enough to prove that for any $l \geq 2$,

$$
\Delta_{l+1}=\Delta_{1} \Delta_{l}-\Delta_{l-1} .
$$

In order to simplify our notation, we denote by LHS the left side of the equality above and by RHS the right side. We thus have

$$
\begin{aligned}
\mathrm{RHS}= & \left(X_{R_{0}^{(p)}}-X_{R_{1}^{(p-2)}}\right)\left(X_{R_{0}^{(l p)}}-X_{R_{1}^{(l p-2)}}\right)-\left(X_{R_{0}^{((l-1) p)}}-X_{R_{1}^{(l-1) p-2)}}\right) \\
= & X_{R_{0}^{(p)}} X_{R_{0}^{(l p)}}-X_{R_{0}^{(p)}} X_{R_{1}^{(l p-2)}}-X_{R_{1}^{(p-2)}} X_{R_{0}^{(l p)}} \\
& \quad+X_{R_{1}^{(p-2)}} X_{R_{1}^{(l p-2)}}-X_{R_{0}^{(l-1) p)}}+X_{R_{1}^{(l-1) p-2)} .}
\end{aligned}
$$

But, according to the multiplication theorem, we get

$$
X_{R_{0}^{(p)}} X_{R_{0}^{(l p)}}=X_{R_{0}^{((l+1) p)}}+X_{R_{0}^{(p-1)}} X_{R_{1}^{(l p-1)},}
$$

so

$$
\begin{aligned}
\text { LHS }=\text { RHS } \Longleftrightarrow & X_{R_{0}^{(p-1)}} X_{R_{1}^{(l p-1)}}-X_{R_{0}^{(p)}} X_{R_{1}^{(l p-2)}}-X_{R_{1}^{(p-2)}} X_{R_{0}^{(l p)}} \\
& \quad+X_{R_{1}^{(p-2)}} X_{R_{1}^{(l p-2)}}-X_{R_{0}^{(l-1) p)}}+X_{R_{1}^{(l-1) p-2)}}+X_{R_{1}^{(l+1) p-2)}}=0 .
\end{aligned}
$$

Applying the multiplication theorem, we get

so

$$
X_{R_{0}^{(l p-2)}} X_{R_{l p-2}^{(p)}}=X_{R_{0}^{((l+1) p-2)}}+X_{R_{0}^{(l p-3)}} X_{R_{l p-1}^{(p-1)}},
$$

$$
X_{R_{1}^{(l+1) p-2)}}=X_{R_{1}^{(l p-2)}} X_{R_{p-1}^{(p)}}-X_{R_{1}^{(l p-3)}} X_{R_{0}^{(p-1)}},
$$

and thus

$$
\begin{aligned}
\text { LHS }=\text { RHS } \Longleftrightarrow & X_{R_{0}^{(p-1)}} X_{R_{1}^{(l p-1)}}-X_{R_{0}^{(p)}} X_{R_{1}^{(l p-2)}}-X_{R_{1}^{(p-2)}} X_{R_{0}^{(l p)}}+X_{R_{1}^{(p-2)}} X_{R_{1}^{(l p-2)}} \\
& -X_{R_{0}^{((l-1) p)}}+X_{R_{1}^{(l-1) p-2)}}+X_{R_{1}^{(l p-2)}} X_{R_{p-1}^{(p)}}-X_{R_{1}^{(l p-3)}} X_{R_{0}^{(p-1)}}=0 .
\end{aligned}
$$


But

$$
X_{R_{0}^{(p)}} X_{R_{1}^{(l p-2)}}=X_{R_{0}^{(l p-1)}} X_{R_{1}^{(p-1)}}+X_{R_{1}^{((l-1) p-2)}}
$$

hence,

$$
\begin{aligned}
\text { LHS }=\text { RHS } \Longleftrightarrow & X_{R_{0}^{(p-1)}} X_{R_{1}^{(l p-1)}}-X_{R_{0}^{(l p-1)}} X_{R_{1}^{(p-1)}} \\
& -X_{R_{1}^{(p-2)}} X_{R_{0}^{(l p)}}+X_{R_{1}^{(p-2)}} X_{R_{1}^{(l p-2)}} \\
& -X_{R_{0}^{((l-1) p)}}+X_{R_{1}^{(l p-2)}} X_{R_{p-1}^{(p)}}-X_{R_{1}^{(l p-3)}} X_{R_{0}^{(p-1)}}=0 \\
\Longleftrightarrow & X_{R_{0}^{(p-1)}} X_{R_{1}^{(l p-1)}}-X_{R_{0}^{(l p-1)}} X_{R_{1}^{(p-1)}} \\
& -X_{R_{1}^{(p-2)}} X_{R_{0}^{(l p)}}-X_{R_{0}^{(l-1) p)}}-X_{R_{1}^{(l p-3)}} X_{R_{0}^{(p-1)}} \\
& +X_{R_{1}^{(l p-2)}}\left(X_{R_{1}^{(p-2)}}+X_{R_{p-1}^{(p)}}\right)=0 .
\end{aligned}
$$

Theorem 2.2 gives

$$
X_{R_{p-1}} X_{R_{0}^{(p-1)}}=X_{R_{1}^{(p-2)}}+X_{R_{p-1}^{(p)}}
$$

so

$$
\begin{aligned}
\text { LHS }=\text { RHS } \Longleftrightarrow X_{R_{0}^{(p-1)}}\left(X_{R_{1}^{(l p-1)}}-X_{R_{1}^{(l p-3)}}+X_{R_{1}^{(l p-2)}} X_{R_{p-1}}\right) \\
\\
\quad-X_{R_{0}^{((l-1) p)}}-X_{R_{0}^{(l p-1)}} X_{R_{1}^{(p-1)}}-X_{R_{1}^{(p-2)}} X_{R_{0}^{(l p)}}=0 .
\end{aligned}
$$

The three-term relation for generalized Chebyshev polynomials gives

$$
X_{R_{1}^{(l p-1)}}=X_{R_{p-1}} X_{R_{1}^{(l p-2)}}-X_{R_{1}^{(l p-3)}}
$$

Thus

LHS $=$ RHS

$$
\Longleftrightarrow 2 X_{R_{0}^{(p-1)}} X_{R_{1}^{(l p-1)}}-\left(X_{R_{0}^{((l-1) p)}}+X_{R_{0}^{(l p-1)}} X_{R_{1}^{(p-1)}}+X_{R_{0}^{(l p)}} X_{R_{1}^{(p-2)}}\right)=0 .
$$

Theorem 2.2 gives

$$
X_{R_{0}^{(p-1)}} X_{R_{1}^{(l p-1)}}=X_{R_{0}^{(l p)}} X_{R_{1}^{(p-2)}}+X_{R_{0}^{((l-1) p)}},
$$

so we finally get

$$
\text { LHS }=\text { RHS } \Longleftrightarrow X_{R_{0}^{(p-1)}} X_{R_{1}^{(l p-1)}}-X_{R_{0}^{(l p-1)}} X_{R_{1}^{(p-1)}}=0 .
$$

The second equality holds by Lemma 3.2, so we have proved that for any $l \geq 2$,

$$
\Delta_{l+1}=\Delta_{1} \Delta_{l}-\Delta_{l-1}
$$

Since we know that $\Delta_{1}=X_{\delta}$ and $\Delta_{2}=F_{2}\left(X_{\delta}\right)$, it follows that $\Delta_{l}=F_{l}\left(X_{\delta}\right)$ for any $l \geq 1$.

We are now able to prove the general difference property: 
Theorem 3.4. Let $Q$ be an affine quiver, $\mathcal{T}$ be a tube of rank $p \geq 1$ in $\Gamma(k Q$-mod $)$. For any $l \geq 1$ and any $0 \leq k \leq p-1$, we have

$$
\mathfrak{b}_{l \delta+\operatorname{dim} R_{0}^{(k)}}=X_{R_{0}^{(k)}} F_{l}\left(X_{\delta}\right)=X_{R_{0}^{(l p+k)}}-X_{R_{k+1}^{(l p-k-2)},}
$$

with the convention that $X_{R_{0}^{(-1)}}=0$.

Proof. The first equality follows from

$$
\operatorname{den}\left(X_{R_{0}^{(k)}} X_{\delta}^{l}\right)=\operatorname{dim} R_{0}^{(k)}+l \delta,
$$

so

$$
\mathfrak{b}_{l \delta+\operatorname{dim} R_{0}^{(k)}}=X_{R_{0}^{(k)}} F_{l}\left(X_{\delta}\right)
$$

We now prove that

$$
X_{R_{0}^{(k)}} F_{l}\left(X_{\delta}\right)=X_{R_{0}^{(l p+k)}}-X_{R_{k+1}^{(l p-k-2)},}
$$

with the convention that $X_{R_{0}^{(-1)}}=0$.

We denote by LHS the left side and by RHS the right side of this equation.

$$
\begin{aligned}
\text { LHS } & =X_{R_{0}^{(k)}}\left(X_{R_{k}^{(l p)}}-X_{R_{k+1}^{(l p-2)}}\right)=X_{R_{0}^{(k)}} X_{R_{k}^{(l p)}}-X_{R_{0}^{(k)}} X_{R_{k+1}^{(l p-2)}} \\
& =X_{R_{0}^{(l p+k)}}+X_{R_{0}^{(k-1)}} X_{R_{k+1}^{(l p-1)}}-X_{R_{0}^{(k)}} X_{R_{k+1}^{(l p-2)}}
\end{aligned}
$$

If $l=1$ and $k=p-1$, we get

$$
\text { LHS }=X_{R_{0}^{(l p+k)}}+X_{R_{0}^{(p-2)}} X_{R_{0}^{(p-1)}}-X_{R_{0}^{(p-1)}} X_{R_{0}^{(p-2)}}=X_{R_{0}^{(l p+k)}}=\text { RHS. }
$$

Otherwise, LHS = RHS if and only if

$$
X_{R_{k+1}^{(l p-k-2)}}=X_{R_{0}^{(k)}} X_{R_{k+1}^{(l p-2)}}-X_{R_{0}^{(k-1)}} X_{R_{k+1}^{(l p-1)}} .
$$

Using the three-term recurrence relations for generalized Chebyshev polynomials, we have

$$
X_{R_{0}^{(k)}}=X_{R_{k-1}} X_{R_{0}^{(k-1)}}-X_{R_{0}^{(k-2)}} \quad \text { and } \quad X_{R_{k+1}^{(l p-1)}}=X_{R_{l p+k-1}} X_{R_{k+1}^{(l p-2)}}-X_{R_{k+1}^{(l p-3)}},
$$

so that, replacing in the right side of (3-2), we get

$$
X_{R_{0}^{(k)}} X_{R_{k+1}^{(l p-2)}}-X_{R_{0}^{(k-1)}} X_{R_{k+1}^{(l p-1)}}=X_{R_{0}^{(k-1)}} X_{R_{k+1}^{(l p-3)}}-X_{R_{0}^{(k-2)}} .
$$

Thus, by induction, we get

$$
X_{R_{0}^{(k)}} X_{R_{k+1}^{(l p-2)}}-X_{R_{0}^{(k-1)}} X_{R_{k+1}^{(l p-1)}}=X_{R_{0}} X_{R_{k+1}^{(l p-k-1)}}-X_{R_{k+1}^{(l p-k)}} .
$$

Now, the three-term recurrence relation gives

$$
X_{R_{k+1}^{(l p-k)}}=X_{R_{k+1+l p-k-1}} X_{R_{k+1}^{(l p-k-1)}}-X_{R_{k+1}^{(l p-k-2)}}=X_{R_{0}} X_{R_{k+1}^{(l p-k-1)}}-X_{R_{k+1}^{(l p-k-2)}},
$$


and thus

$$
X_{R_{0}} X_{R_{k+1}^{(l p-k-1)}}-X_{R_{k+1}^{(l p-k)}}=X_{R_{k+1}^{(l p-k-2)}}
$$

proving that (3-2) holds.

As a corollary, for any positive root $\boldsymbol{d}$ with defect zero, we obtain a description of $\mathfrak{b}_{\boldsymbol{d}}$ as a certain difference of cluster characters:

Corollary 3.5. Let $Q$ be an affine quiver and $\boldsymbol{d}$ be a positive root with defect zero. Let $M$ be any indecomposable representation of dimension $\boldsymbol{d}$. Then there exists a quasisimple module $R_{0}$ in a tube of rank $p \geq 1$, an integer $0 \leq k \leq p-1$, and an integer $l \geq 0$ such that $\boldsymbol{d}=l \delta+\operatorname{dim} R_{0}^{(k)}$. Moreover, for any such $R_{0}, k, l$, we have

$$
\mathfrak{b}_{d}=X_{R_{0}^{(k)}} F_{l}\left(X_{\delta}\right)=X_{R_{0}^{(l p+k)}}-X_{R_{k+1}^{(l p-k-2)},}
$$

where $R_{i}$, with $i \in \mathbb{Z} / p \mathbb{Z}$, are the quasisimple modules in $\mathcal{T}$ ordered such that $\tau R_{i} \simeq R_{i-1}$ for every $i \in \mathbb{Z} / p \mathbb{Z}$.

\section{Integrable bundles on $\operatorname{rep}_{k}(Q)$ and their characters}

In the previous section, we obtained a realization of the elements $\mathfrak{b}_{d}$ associated to defect zero roots as differences of cluster characters. The aim of this section is to introduce a new map $\theta_{\operatorname{Tr}}$ such that these elements correspond precisely to values of $\theta_{\mathrm{Tr}}$.

Unless otherwise specified, $Q$ denotes an arbitrary acyclic quiver in this section.

Integrable bundles. For any $\boldsymbol{d} \in \mathbb{N}^{Q_{0}}$, the representation variety $\operatorname{rep}_{\boldsymbol{k}}(Q, \boldsymbol{d})$ of dimension $\boldsymbol{d}$ is the set of all representations $M$ of $Q$ with dimension vector $\boldsymbol{d}$. Note that

$$
\operatorname{rep}_{\boldsymbol{k}}(Q, \boldsymbol{d}) \simeq \prod_{i \rightarrow j \in Q_{1}} \operatorname{Hom}_{\boldsymbol{k}}\left(\boldsymbol{k}^{d_{i}}, \boldsymbol{k}^{d_{j}}\right)
$$

so that $\operatorname{rep}_{\boldsymbol{k}}(Q, \boldsymbol{d})$ is an affine irreducible variety.

Definition 4.1. Let $Q$ be any acyclic quiver. An integrable bundle on $\operatorname{rep}_{\boldsymbol{k}}(Q)$ is a map

$$
\mathscr{F}: M \mapsto \mathscr{F}(M) \subset \operatorname{Gr}(M)
$$

defined on the set of indecomposable objects in $\operatorname{rep}_{k}(Q)$ such that for any $M \in$ $\operatorname{rep}_{k}(Q)$,

- for any $\boldsymbol{e} \in \mathbb{N}^{Q_{0}}, \mathscr{F}_{\boldsymbol{e}}(M)=\mathscr{F}_{\boldsymbol{F}}(M) \cap \mathrm{Gr}_{\boldsymbol{e}}(M)$ is constructible, and

- if $M \simeq N$ in $\operatorname{rep}_{\boldsymbol{k}}(Q)$, then $\chi\left(\mathscr{F}_{\boldsymbol{e}}(M)\right) \simeq \chi\left(\mathscr{F}_{\boldsymbol{e}}(N)\right)$ for any $\boldsymbol{e} \in \mathbb{N} Q_{0}$. 
Remark 4.2. Note that, if $\mathscr{F}$ is an integrable bundle on $\operatorname{rep}_{k}(Q)$, then the family $\left(\chi\left(\mathscr{F}_{\boldsymbol{e}}(M)\right)\right)_{\boldsymbol{e} \in \mathbb{N} Q_{0}}$ has finite support.

Example 4.3. The map $M \mapsto \operatorname{Gr}(M)$ is an integrable bundle called the quiver Grassmannian bundle.

For any $\boldsymbol{k} Q$-module $M$ and any submodule $U \subset M$, we set

$$
\operatorname{Gr}^{U}(M)=\{N \in \operatorname{Gr}(M) \mid U \text { is a submodule of } N\} .
$$

This is a constructible subset in the quiver Grassmannian $\operatorname{Gr}(M)$.

If $Q$ is an affine quiver, we define another integrable bundle $\operatorname{Tr}$ as follows. Let $M$ be an indecomposable $\boldsymbol{k} Q$-module. If $M$ is rigid, we set $\operatorname{Tr}(M)=\operatorname{Gr}(M)$. If $M$ is not rigid, it is regular and we can thus write $M=R_{0}^{(l p+k)}$ for some quasisimple module $R_{0}$ in a tube of rank $p \geq 1, l \geq 1$ and $0 \leq k \leq p-1$. There exists a nonzero monomorphism $\imath: R_{0}^{(l p-1)} \rightarrow R_{0}^{(l p)}$ such that

$$
\operatorname{Hom}_{k Q}\left(R_{0}^{(l p-1)}, R_{0}^{(l p)}\right) \simeq \boldsymbol{k} l .
$$

The set $l\left(\mathrm{Gr}^{R_{0}^{(k+1)}}\left(R_{0}^{(l p-1)}\right)\right)$ is a constructible subset of $\operatorname{Gr}\left(R_{0}^{(l p)}\right)$, and since

$$
\operatorname{Hom}_{\boldsymbol{k} Q}\left(R_{0}^{(l p-1)}, R_{0}^{(l p)}\right) \simeq \boldsymbol{k} \boldsymbol{l},
$$

it does not depend on the choice of $l$. We can thus identify $\mathrm{Gr}^{R_{0}^{(k+1)}}\left(R_{0}^{(l p-1)}\right)$ with a constructible subset of $\operatorname{Gr}\left(R_{0}^{(l p)}\right)$. With these notations and identifications, we set

$$
\operatorname{Tr}(M)=\operatorname{Gr}(M) \backslash \mathrm{Gr}^{R^{(k+1)}}\left(R_{0}^{(l p-1)}\right) .
$$

Note that if $l=0, M$ is rigid and we recover the equality $\operatorname{Tr}(M)=\operatorname{Gr}(M)$.

For every dimension vector $\boldsymbol{e} \in \mathbb{N}^{Q_{0}}$ and any indecomposable $\boldsymbol{k} Q$-module $M$, the transverse quiver Grassmannian of $M$ (of dimension $\boldsymbol{e}$ ) is the constructible subset of $\mathrm{Gr}_{e}(M)$

$$
\operatorname{Tr}_{\boldsymbol{e}}(M)=\{N \in \operatorname{Tr}(M) \mid \operatorname{dim} N=\boldsymbol{e}\} .
$$

The map

$$
\operatorname{Tr}: M \mapsto \operatorname{Tr}(M) \subset \operatorname{Gr}(M)
$$

is an integrable bundle on $\operatorname{rep}_{k}(Q)$.

Character associated to an integrable bundle. Extending an idea of Caldero and Chapoton, we associate to any integrable bundle on $\operatorname{rep}_{k}(Q)$ a map from the set of objects in $\mathscr{C}_{Q}$ to the ring $\mathbb{Z}\left[\boldsymbol{u}^{ \pm 1}\right]$ of Laurent polynomials in the initial cluster of $A(Q)$.

Definition 4.4. Let $\mathscr{F}$ be an integrable bundle on $\operatorname{rep}_{k}(Q)$. The character associated to $\mathscr{F}$ is the map

$$
\theta_{\mathscr{F}}(?): \mathrm{Ob}\left(\mathscr{C}_{Q}\right) \rightarrow \mathbb{Z}\left[\boldsymbol{u}^{ \pm 1}\right]
$$

given by: 
- If $M \simeq P_{i}[1]$ for some $i \in Q_{0}$, then $\theta_{\mathscr{F}}\left(P_{i}[1]\right)=u_{i}$.

- If $M$ is an indecomposable $\boldsymbol{k} Q$-module, then

$$
\theta_{\mathscr{F}}(M)=\sum_{\boldsymbol{e} \in \mathbb{N} Q_{0}} \chi\left(\mathscr{F}_{\boldsymbol{e}}(M)\right) \prod_{i \in Q_{0}} u_{i}^{-\left\langle\boldsymbol{e}, S_{i}\right\rangle-\left\langle S_{i}, \operatorname{dim} M-\boldsymbol{e}\right\rangle} .
$$

- $\theta_{\mathscr{F}}(M \oplus N)=\theta_{\mathscr{F}}(M) \theta_{\mathscr{F}}(N)$ for any two objects $M, N$ in $\mathscr{b}_{Q}$.

We now prove that $\theta_{\operatorname{Tr}}$ coincides with $X_{\text {? }}$ on the set of rigid objects in $\mathscr{C}_{Q}$. In particular, this will allow us to realize cluster monomials in terms of $\theta_{\mathrm{Tr}}$.

Lemma 4.5. Let $Q$ be an affine quiver. Then, for any rigid object $M$ in $\mathscr{C}_{Q}$, we have $\theta_{\operatorname{Tr}}(M)=X_{M}$. In particular,

$$
M(Q)=\left\{\theta_{\operatorname{Tr}}(M) \mid M \text { is rigid in } \mathscr{C}_{Q}\right\} .
$$

Proof. Let $M$ be a rigid object in $\mathscr{C}_{Q}$. We write

$$
M=P_{i_{1}}[1] \oplus \cdots \oplus P_{i_{r}}[1] \oplus M_{1} \oplus \cdots \oplus M_{s},
$$

where each $P_{i_{j}}$ is an indecomposable projective $k Q$-module and each $M_{i}$ is an indecomposable module. Also, since $M$ is rigid, each $M_{i}$ is a rigid $\boldsymbol{k} Q$-module and thus $\operatorname{Tr}\left(M_{i}\right)=\operatorname{Gr}\left(M_{i}\right)$ for any $i \in\{1, \ldots, s\}$. In particular, it follows that $\theta_{\operatorname{Tr}}\left(M_{i}\right)=X_{M_{i}}$ for any $i \in\{1, \ldots, s\}$. Then

$$
\begin{aligned}
\theta_{\operatorname{Tr}}(M) & =\theta_{\operatorname{Tr}}\left(P_{i_{1}}[1] \oplus \cdots \oplus P_{i_{r}}[1] \oplus M_{1} \oplus \cdots \oplus M_{s}\right) \\
& =\theta_{\operatorname{Tr}}\left(P_{i_{1}}[1]\right) \cdots \theta_{\operatorname{Tr}}\left(P_{i_{r}}[1]\right) \theta_{\operatorname{Tr}}\left(M_{1}\right) \cdots \theta_{\operatorname{Tr}}\left(M_{s}\right) \\
& =u_{i_{1}} \cdots u_{i_{r}} X_{M_{1}} \cdots X_{M_{s}} \\
& =X_{P_{i_{1}}[1] \oplus \cdots \oplus P_{i_{r}}[1] \oplus M_{1} \oplus \cdots \oplus M_{s}}=X_{M} .
\end{aligned}
$$

The second assertion follows directly from Caldero and Keller's realization of cluster monomials:

$$
\mathcal{M}(Q)=\left\{X_{M} \mid M \text { is rigid in } \mathscr{C}_{Q}\right\}=\left\{\theta_{\operatorname{Tr}}(M) \mid M \text { is rigid in } \mathscr{C}_{Q}\right\} .
$$

\section{A geometrization of $\mathscr{B}(Q)$}

We now relate the character $\theta_{\operatorname{Tr}}$ with the difference properties obtained in Section 3. This will provide a realization of the elements in $\mathscr{B}(Q)$ in terms of $\theta_{\mathrm{Tr}}$.

From difference properties to $\boldsymbol{\theta}_{\mathrm{Tr}}$. Using Theorem 3.4, we first deduce a realization in terms of $\theta_{\operatorname{Tr}}$ of the elements in $\mathscr{B}(Q)$ corresponding to positive roots.

Theorem 5.1. Let $\boldsymbol{d}$ be any positive root. Then $\mathfrak{b}_{\boldsymbol{d}}=\theta_{\mathrm{Tr}}(M)$, where $M$ is any indecomposable representation of dimension $\boldsymbol{d}$. 
Proof. If $\boldsymbol{d}$ is a positive root with nonzero defect, then $\boldsymbol{d}$ is real and there exists a unique indecomposable representation $M$ in $\operatorname{rep}_{k}(Q, d)$. Moreover, this representation has to be preprojective or preinjective. In both cases, it is rigid and thus $\mathfrak{b}_{\boldsymbol{d}}=X_{M}=\theta_{\operatorname{Tr}}(M)$. We can thus assume that $\boldsymbol{d} \in \mathbb{N}^{Q_{0}}$ is a root with defect zero.

Let $M$ be an indecomposable representation in $\operatorname{rep}_{k}(Q, d)$. It is necessarily contained in a tube $\mathcal{T}$ of rank $p \geq 1$. We denote by $R_{i}$, with $i \in \mathbb{Z} / p \mathbb{Z}$, the quasisimple modules in $\mathscr{T}$ ordered such that $\tau R_{i} \simeq R_{i-1}$ for any $i \in \mathbb{Z} / p \mathbb{Z}$. We can write $\boldsymbol{d}=l \delta+\boldsymbol{n}$, where $\boldsymbol{n}$ is either a real Schur root or zero. If $\boldsymbol{n} \neq 0$, there exists a unique indecomposable representation $N$ in $\operatorname{rep}_{\boldsymbol{k}}(Q, \boldsymbol{n})$. In any case, if $M \simeq R_{0}^{(l p+k)}$ with $l \geq 0$ and $0 \leq k \leq p-1, N$ is the rigid representation $R_{0}^{(k)}$ (still with the convention that $R_{0}^{(0)}=0$ ) and

$$
\mathfrak{b}_{d}=X_{R_{0}^{(k)}} F_{l}\left(X_{\delta}\right)
$$

Now, according to Theorem 3.4, we have

$$
X_{R_{0}^{(k)}} F_{l}\left(X_{\delta}\right)=X_{R_{0}^{(l p+k)}}-X_{R_{k+1}^{(l p-k-2)}} .
$$

For any $\boldsymbol{e} \in \mathbb{N}^{Q_{0}}$, the map $\operatorname{Gr}_{\boldsymbol{e}}^{R_{0}^{(k+1)}}\left(R_{0}^{(l p-1)}\right) \rightarrow \operatorname{Gr}_{\boldsymbol{e}-\operatorname{dim} R_{0}^{(k+1)}}\left(R_{k+1}^{(l p-k-2)}\right)$ given by

$$
U \mapsto U / R_{0}^{(k+1)}
$$

is an algebraic isomorphism, and we denote by $c_{\boldsymbol{e}} \in \mathbb{Z}$ the common value of the Euler characteristics of these constructible sets. Fix now some $\boldsymbol{e} \in \mathbb{N} Q_{0}$; the monomial corresponding to $\boldsymbol{e}$ in $X_{R_{0}^{(l p+k)}}$ is

$$
c_{e} \prod_{i} u_{i}^{-\left\langle e, S_{i}\right\rangle-\left\langle S_{i}, \operatorname{dim} R_{0}^{(l p+k)}-e\right\rangle},
$$

and the monomial corresponding to $\boldsymbol{e}-\operatorname{dim} R_{0}^{(k+1)}$ in $X_{R_{k+1}^{(p p-k-2)}}$ is

$$
c_{\boldsymbol{e}} \prod_{i} u_{i}^{-\left\langle\boldsymbol{e}-\operatorname{dim} R_{0}^{(k+1)}, S_{i}\right\rangle-\left\langle S_{i}, \operatorname{dim} R_{k+1}^{(l p-k-2)}+\operatorname{dim} R_{0}^{(k+1)}-\boldsymbol{e}\right\rangle} .
$$

We now prove that these monomials are the same. For any $i=0, \ldots, p-1$, we set $r_{i}=\operatorname{dim} R_{i}$ and we denote by $c$ the Coxeter transformation on $\mathbb{Z}^{Q_{0}}$ induced by the Auslander-Reiten translation. We recall that for any $\beta, \gamma \in \mathbb{Z}^{Q_{0}}$, we have $\langle\gamma, c(\beta)\rangle=-\langle\beta, \gamma\rangle$. With this notation, we have

$$
\operatorname{dim} R_{0}^{(k+1)}=r_{0}+\cdots+r_{k} \quad \text { and } \quad \operatorname{dim} R_{k+1}^{(l p-k-2)}=(l-1) \delta+r_{k+1}+\cdots r_{p-2},
$$

$$
\operatorname{dim} R_{0}^{(k+1)}+\operatorname{dim} R_{k+1}^{(l p-k-2)}=l \delta-r_{p-1}
$$


We now compute the exponents:

$$
\begin{aligned}
-\left\langle\boldsymbol{e}, S_{i}\right\rangle-\left\langle S_{i}, \operatorname{dim} R_{0}^{(l p+k)}-\boldsymbol{e}\right\rangle & =-\left\langle\boldsymbol{e}, S_{i}\right\rangle-\left\langle S_{i}, l \delta+r_{0}+\cdots+r_{k-1}-\boldsymbol{e}\right\rangle \\
& =-\left\langle\boldsymbol{e}, S_{i}\right\rangle-\left\langle S_{i}, l \delta-\boldsymbol{e}\right\rangle-\left\langle S_{i}, r_{0}+\cdots+r_{k-1}\right\rangle \\
& =-\left\langle\boldsymbol{e}, S_{i}\right\rangle-\left\langle S_{i}, l \delta-\boldsymbol{e}\right\rangle-\left\langle r_{1}+\cdots+r_{k}, S_{i}\right\rangle
\end{aligned}
$$

$-\left\langle e-\operatorname{dim} R_{0}^{(k+1)}, S_{i}\right\rangle-\left\langle S_{i}, \operatorname{dim} R_{k+1}^{(l p-k-2)}+\operatorname{dim} R_{0}^{(k+1)}-e\right\rangle$

$$
\begin{aligned}
& =-\left\langle\boldsymbol{e}, S_{i}\right\rangle+\left\langle r_{0}+\cdots+r_{k}, S_{i}\right\rangle+\left\langle S_{i}, r_{p-1}\right\rangle-\left\langle S_{i}, l \delta-\boldsymbol{e}\right\rangle \\
& =-\left\langle\boldsymbol{e}, S_{i}\right\rangle-\left\langle S_{i}, l \delta-\boldsymbol{e}\right\rangle+\left\langle r_{1}+\cdots+r_{k}, S_{i}\right\rangle .
\end{aligned}
$$

Thus the two monomials are the same. It follows that

$$
\begin{aligned}
& X_{R_{0}^{(l p+k)}}-X_{R_{k+1}^{(l p-k-2)}} \\
& =\sum_{\boldsymbol{e}} \chi\left(\operatorname{Gr}_{\boldsymbol{e}}\left(R_{0}^{(l p+k)}\right)\right) \prod_{i} u_{i}^{-\left\langle\boldsymbol{e}, S_{i}\right\rangle-\left\langle S_{i}, l \delta-\boldsymbol{e}\right\rangle-\left\langle r_{1}+\cdots+r_{k}, S_{i}\right\rangle} \\
& -\sum_{\boldsymbol{e}} \chi\left(\mathrm{Gr}_{\boldsymbol{e}-\operatorname{dim} R_{0}^{(k+1)}}\left(R_{k+1}^{(l p-k-2)}\right)\right) \prod_{i} u_{i}^{-\left\langle\boldsymbol{e}, S_{i}\right\rangle-\left\langle S_{i}, l \delta-\boldsymbol{e}\right\rangle-\left\langle r_{1}+\cdots+r_{k}, S_{i}\right\rangle} \\
& =\sum_{\boldsymbol{e}} \chi\left(\operatorname{Gr}_{\boldsymbol{e}}\left(R_{0}^{(l p+k)}\right) \backslash \operatorname{Gr}_{\boldsymbol{e}}^{R_{0}^{(k+1)}}\left(R_{0}^{(l p-1)}\right)\right) \prod_{i} u_{i}^{-\left\langle\boldsymbol{e}, S_{i}\right\rangle-\left\langle S_{i}, l \delta-\boldsymbol{e}\right\rangle-\left\langle r_{1}+\cdots+r_{k}, S_{i}\right\rangle} \\
& =\sum_{\boldsymbol{e}} \chi\left(\operatorname{Tr}_{\boldsymbol{e}}\left(R_{0}^{(l p+k)}\right)\right) \prod_{i} u_{i}^{-\left\langle\boldsymbol{e}, S_{i}\right\rangle-\left\langle S_{i}, \operatorname{dim} R_{0}^{(l p+k)}-\boldsymbol{e}\right\rangle} .
\end{aligned}
$$

Realization of $\mathscr{B}(\boldsymbol{Q})$ in terms of $\boldsymbol{\theta}_{\mathrm{Tr}}$. Summing up the previous results, we deduce the following geometric description of $\mathscr{B}(Q)$ :

Theorem 5.2. Let $Q$ be an affine quiver. Then

$\mathscr{B}(Q)=\left\{\begin{array}{l|l}\theta_{\operatorname{Tr}}(M \oplus R) & \begin{array}{l}M \text { is an indecomposable (or zero) regular } \boldsymbol{k} Q \text {-module, } \\ R \text { is any rigid object in } \mathscr{C}_{Q} \text { such that } \operatorname{Ext}_{\mathscr{C}_{Q}}^{1}(M, R)=0\end{array}\end{array}\right.$.

Proof. We denote by $\mathscr{S}$ the right side of the claimed equality. By definition,

$$
\mathscr{B}(Q)=M(Q) \sqcup\left\{F_{l}\left(X_{\delta}\right) X_{R} \mid l \geq 1, R \text { is a regular rigid } \boldsymbol{k} Q \text {-module }\right\}
$$

We first prove that $\mathscr{S} \subset \mathscr{B}(Q)$. Let $R$ be a rigid object in $\mathscr{C}_{Q}$; then $\theta_{\operatorname{Tr}}(R)$ is a cluster monomial by Lemma 4.5. Fix now $M$ to be an indecomposable regular $\boldsymbol{k} Q$-module in a tube $\mathscr{T}$ such that $\operatorname{Ext}_{\mathscr{C}_{Q}}^{1}(M, R)=0$. If $M$ is rigid, then $M \oplus R$ is rigid in $\mathscr{C}_{Q}$, and $\theta_{\operatorname{Tr}}(M) \theta_{\operatorname{Tr}}(R)=\theta_{\operatorname{Tr}}(M \oplus R)$ is a cluster monomial by Lemma 4.5. Now, if $M$ is nonrigid, then $\boldsymbol{d}=\operatorname{dim} M$ is a positive root of defect zero, and thus $\theta_{\operatorname{Tr}}(M)=\mathfrak{b}_{\boldsymbol{d}}$ by Theorem 5.1. Thus, there exists $l \geq 1$ and $N$ an indecomposable 
rigid (or zero) module in $\mathscr{T}$ such that $\boldsymbol{d}=l \delta+\operatorname{dim} N$. According to Theorem 5.1,

$$
\begin{aligned}
\theta_{\operatorname{Tr}}(M \oplus R) & =\theta_{\operatorname{Tr}}(M) \theta_{\operatorname{Tr}}(R)=\mathfrak{b}_{l \delta+\operatorname{dim} N} \theta_{\operatorname{Tr}}(R)=F_{l}\left(X_{\delta}\right) X_{N} \theta_{\operatorname{Tr}}(R) \\
& =F_{l}\left(X_{\delta}\right) \theta_{\operatorname{Tr}}(N) \theta_{\operatorname{Tr}}(R)=F_{l}\left(X_{\delta}\right) \theta_{\operatorname{Tr}}(N \oplus R) .
\end{aligned}
$$

Since $\operatorname{Ext}_{\mathscr{C}_{Q}}^{1}(M, R)=0$, we have $\operatorname{Ext}_{k Q}^{1}(M, R)=0$ and $\operatorname{Ext}_{k Q}^{1}(R, M)=0$. Thus, it follows easily that $\operatorname{Ext}_{k Q}^{1}(N, R)=0$ and $\operatorname{Ext}_{k Q}^{1}(R, N)=0$, so $N \oplus R$ is a rigid regular $\boldsymbol{k} Q$-module. In particular, $\theta_{\operatorname{Tr}}(N \oplus R)=X_{N \oplus R}$ and thus

$$
\theta_{\operatorname{Tr}}(M \oplus R)=F_{l}\left(X_{\delta}\right) X_{N \oplus R} \in \mathscr{B}(Q) .
$$

Conversely, fix an element in $\mathscr{B}(Q)$. If $x$ is a cluster monomial, then according to Lemma 4.5, there exists some rigid object $M$ in $\mathscr{C}_{Q}$ such that $x=\theta_{\operatorname{Tr}}(M)$. Thus, $x \in \mathscr{Y}$. Fix now some regular rigid $\boldsymbol{k} Q$-module $R$ and some integer $l \geq 1$. Then the direct summands of $R$ belong to exceptional tubes. We fix an indecomposable $\boldsymbol{k} Q$ module $M$ of dimension vector $l \delta$ in a homogeneous tube. $\operatorname{Then}^{\operatorname{Ext}_{\mathscr{C}_{Q}}^{1}}(M, R)=0$. According to Theorem 5.1, we have $F_{l}\left(X_{\delta}\right) X_{R}=\theta_{\operatorname{Tr}}(M) X_{R}$, but $R$ is rigid, so $X_{R}=\theta_{\mathrm{Tr}}(R)$. Thus,

$$
F_{l}\left(X_{\delta}\right) X_{R}=\theta_{\operatorname{Tr}}(M) \theta_{\operatorname{Tr}}(R)=\theta_{\operatorname{Tr}}(M \oplus R) \in \mathscr{S} .
$$

\section{Examples}

We shall now study two examples corresponding to cases where it is known that $\mathscr{B}(Q)$ is the canonically positive basis in $\mathscr{A}(Q)$.

The $\tilde{A}_{1,1}$ case. Let $Q$ be the Kronecker quiver, that is, the affine quiver of type $\tilde{\mathbb{A}}_{1,1}$ with the orientation

$$
Q: \quad 1 \Longrightarrow 2
$$

and minimal imaginary root $\delta=(11)$.

For any $\lambda \in \boldsymbol{k}$, we set

$$
M_{\lambda}: \quad \boldsymbol{k} \underset{\lambda}{\stackrel{1}{\longrightarrow}} \boldsymbol{k}
$$

and

$$
M_{\infty}: \quad \boldsymbol{k} \underset{1}{\stackrel{0}{\longrightarrow}} \boldsymbol{k} .
$$

It is well-known that every tube in $\Gamma(\boldsymbol{k} Q$-mod $)$ is homogeneous and that the family $\left\{M_{\lambda} \mid \lambda \in \boldsymbol{k} \sqcup\{\infty\}\right\}$ is a complete set of representatives of pairwise nonisomorphic quasisimple $\boldsymbol{k} Q$-modules.

For any $n \geq 1$, the indecomposable representations of quasilength $n$ are given by

$$
M_{\lambda}^{(n)}: \quad \boldsymbol{k}^{n} \underset{J_{n}(\lambda)}{\stackrel{1}{\longrightarrow}} \boldsymbol{k}^{n}
$$


for any $\lambda \in \boldsymbol{k}$ and

$$
M_{\infty}^{(n)}: \quad \boldsymbol{k}^{n} \underset{1}{\stackrel{J_{n}(0)}{\rightleftarrows}} \boldsymbol{k}^{n}
$$

where $J_{n}(\lambda) \in M_{n}(\boldsymbol{k})$ denotes the Jordan block of size $n$ associated to the eigenvalue $\lambda$. Quiver Grassmannians and transverse quiver Grassmannians of indecomposable representations with quasilength 2 are described in Table 1.

Note that $\boldsymbol{k} Q$-mod contains no regular rigid modules. It follows that in this case

$$
\mathscr{B}(Q)=\mathcal{M}(Q) \sqcup\left\{\theta_{\operatorname{Tr}}(M) \mid M \text { is an indecomposable regular } \boldsymbol{k} Q \text {-module }\right\} .
$$

According to [Sherman and Zelevinsky 2004], this set is the canonically positive basis of $\mathscr{A}(Q)$.

From Table 1, we see that for any $\lambda \in \boldsymbol{k} \sqcup\{\infty\}$,

$$
\theta_{\mathrm{Tr}}\left(M_{\lambda}^{(2)}\right)=\theta_{\mathrm{Gr}}\left(M_{\lambda}^{(2)}\right)-1=X_{M_{\lambda}^{(2)}}-1=S_{2}\left(X_{M_{\lambda}}\right)-1=F_{2}\left(X_{M_{\lambda}}\right)=\mathfrak{b}_{2 \delta} .
$$

This illustrates Theorem 5.1.

The $\tilde{\mathbb{A}}_{2,1}$ case. We now consider the quiver $Q$ of affine type $\tilde{\mathbb{A}}_{2,1}$ equipped with the orientation

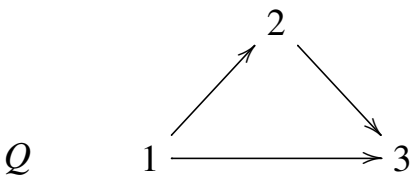

\begin{tabular}{|c|cc|c|}
\hline $\boldsymbol{e}$ & $\operatorname{Gr}_{\boldsymbol{e}}\left(M_{\lambda}^{(2)}\right)$ & $\operatorname{Tr}_{\boldsymbol{e}}\left(M_{\lambda}^{(2)}\right)$ & $\boldsymbol{u}^{\left\langle-\boldsymbol{e}, S_{i}\right\rangle-\left\langle S_{i}, 2 \delta-\boldsymbol{e}\right\rangle}$ \\
\hline$(00)$ & $\{0\}$ & $\{0\}$ & $u_{1}^{2} / u_{2}^{2}$ \\
$(01)$ & $\mathbb{P}^{1} \times\left\{S_{2}\right\}$ & $\mathbb{P}^{1} \times\left\{S_{2}\right\}$ & $1 / u_{2}^{2}$ \\
$(02)$ & $\left\{S_{2} \oplus S_{2}\right\}$ & $\left\{S_{2} \oplus S_{2}\right\}$ & $1 /\left(u_{1}^{2} u_{2}^{2}\right)$ \\
$(11)$ & $\left\{M_{\lambda}\right\}$ & $\varnothing$ & 1 \\
$(12)$ & \multicolumn{2}{|c|}{ see caption } & $1 / u_{1}^{2}$ \\
$(22)$ & $\left\{M_{\lambda}^{(2)}\right\}$ & $\left\{M_{\lambda}^{(2)}\right\}$ & $u_{2}^{2} / u_{1}^{2}$ \\
\hline
\end{tabular}

Table 1. Grassmannians and transverse Grassmannians of indecomposable modules of quasilength 2 in type $\tilde{\mathbb{A}}_{1,1}$. Here $\lambda$ takes values in $\boldsymbol{k} \cup\{0, \infty\}$. For $e=(12)$, the values of both $\operatorname{Gr}_{(12)}\left(M_{\lambda}^{(2)}\right)$ and $\operatorname{Tr}_{(12)}\left(M_{\lambda}^{(2)}\right)$ are $\left\{P_{1}, M_{\lambda} \oplus S_{2}\right\}$ if $\lambda \in\{0, \infty\}$, and $\mathbb{P}^{1} \times\left\{M_{\lambda} \oplus S_{2}\right\}$ otherwise. 
The minimal imaginary root of $Q$ is $\delta=(111)$. For any $\lambda \in \boldsymbol{k}$, we set

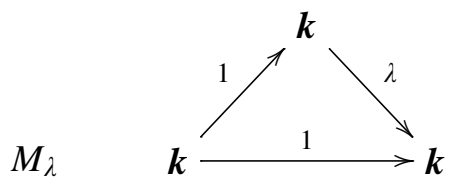

and

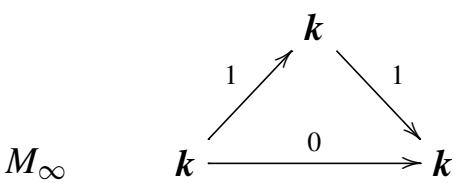

Further, $\Gamma(\boldsymbol{k} Q$-mod) contains exactly one exceptional tube $\mathscr{T}$ of rank 2 , whose quasisimples are

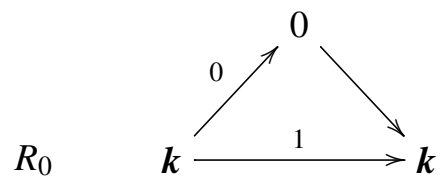

and

$$
R_{1} \simeq S_{2}
$$

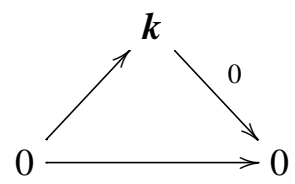

The set $\left\{M_{\lambda} \mid \lambda \in \boldsymbol{k} \sqcup\{\infty\}\right\} \sqcup\left\{R_{0}^{(2)}\right\}$ is a complete set of representatives of pairwise nonisomorphic indecomposable representations in $\operatorname{rep}_{k}(Q, \delta)$. For any $\lambda \neq$ $0, \infty, M_{\lambda}$ is a quasisimple $\boldsymbol{k} Q$-module in a homogeneous tube. Moreover, $M_{0}=$ $R_{1}^{(2)}$ and $M_{\infty}$ is quasisimple in a homogeneous tube.

Quiver Grassmannians and transverse quiver Grassmannians of indecomposable representations of dimension $\delta$ are described in Table 2. For simplicity, we only listed the dimension vectors that lead to nonempty quiver Grassmannians.

In Table 2, we observe that $X_{M_{\lambda}}=X_{M_{0}}-1=X_{M_{\infty}}-1$, illustrating Theorem 3.1. Also, we see that $\theta_{\operatorname{Tr}}\left(M_{\lambda}\right)=\theta_{\operatorname{Tr}}\left(M_{0}\right)=\theta_{\operatorname{Tr}}\left(M_{\infty}\right)$ for any $\lambda \in \boldsymbol{k} \backslash\{0\}$ so that the transverse character does not depend on the chosen tube. Moreover,

$$
\theta_{\operatorname{Tr}}\left(M_{\lambda}\right)=X_{M_{\lambda}}=F_{1}\left(X_{\delta}\right),
$$

illustrating Theorem 5.1.

Remark 6.1. Table 2 justifies the terminology transverse submodule. Indeed, we see that, given two indecomposable regular modules $M$ and $N$ having the same dimension vectors, the submodules $U$ in $\operatorname{Tr}(M)$ are those having a corresponding submodule in $\operatorname{Gr}(N)$. In some sense, we can see $U$ as a submodule "common" to $M$ and $N$. This is why we call it transverse. 


\begin{tabular}{|c|cc|cc|c|}
\hline $\boldsymbol{e}$ & $\operatorname{Gr}_{\boldsymbol{e}}\left(M_{\lambda}\right)$ & $\operatorname{Tr}_{\boldsymbol{e}}\left(M_{\lambda}\right)$ & $\mathrm{Gr}_{\boldsymbol{e}}\left(R_{0}^{(2)}\right)$ & $\operatorname{Tr}_{\boldsymbol{e}}\left(R_{0}^{(2)}\right)$ & $\boldsymbol{u}^{\left\langle-\boldsymbol{e}, S_{i}\right\rangle-\left\langle S_{i}, \delta-\boldsymbol{e}\right\rangle}$ \\
\hline$(000)$ & $\{0\}$ & $\{0\}$ & $\{0\}$ & $\{0\}$ & $u_{1} / u_{3}$ \\
$(001)$ & $\left\{S_{3}\right\}$ & $\left\{S_{3}\right\}$ & $\left\{S_{3}\right\}$ & $\left\{S_{3}\right\}$ & $1 /\left(u_{2} u_{3}\right)$ \\
$(010)$ & see caption & $\varnothing$ & $\varnothing$ & 1 \\
$(011)$ & \multicolumn{2}{|c|}{ see caption } & $\left\{P_{2}\right\}$ & $\left\{P_{2}\right\}$ & $1 /\left(u_{1} u_{2}\right)$ \\
$(101)$ & $\varnothing$ & $\varnothing$ & $\left\{R_{0}\right\}$ & $\varnothing$ & 1 \\
$(111)$ & $\left\{M_{\lambda}\right\}$ & $\left\{M_{\lambda}\right\}$ & $\left\{R_{0}^{(2)}\right\}$ & $\left\{R_{0}^{(2)}\right\}$ & $u_{3} / u_{1}$ \\
\hline
\end{tabular}

Table 2. Grassmannians and transverse Grassmannians for quasilength 2 in type $\tilde{A}_{2,1}$. Here $\lambda$ takes values in $\boldsymbol{k} \cup\{0, \infty\}$. For $e=(010)$, all entries are $\varnothing$ except that $\mathrm{Gr}_{010}\left(M_{0}\right)=\left\{S_{2}\right\}$. For $e=(011)$, all entries equal $\left\{P_{2}\right\}$, apart from that $\operatorname{Gr}_{(011)}\left(M_{0}\right)$ and $\operatorname{Tr}_{(011)}\left(M_{0}\right)$, which equal $\left\{S_{2} \oplus S_{3}\right\}$.

As suggested by Bernhard Keller, this notion of transversality should have a more precise meaning in the context of deformation theory. Some connections are known at this time, and this will be discussed in a forthcoming article.

\section{Acknowledgements}

This paper was written while the author was at the university of Sherbrooke as a CRM-ISM postdoctoral fellow under the supervision of the Ibrahim Assem, Thomas Brüstle and Virginie Charette. He would like to thank Giovanni Cerulli Irelli for motivating the investigation of higher-difference properties during his stay at the University of Padova in June 2009. This was the starting point of this work. He would also like to thank the rest of the algebra group of Padova for their kind hospitality. Finally, he would like to thank Bernhard Keller, Philippe Caldero and Frédéric Chapoton for interesting discussions on the topic.

\section{References}

[Berenstein et al. 2005] A. Berenstein, S. Fomin, and A. Zelevinsky, "Cluster algebras, III: Upper bounds and double Bruhat cells", Duke Math. J. 126:1 (2005), 1-52. MR 2005i:16065 Zbl 1135. 16013

[Buan et al. 2006] A. B. Buan, R. Marsh, M. Reineke, I. Reiten, and G. Todorov, "Tilting theory and cluster combinatorics", Adv. Math. 204:2 (2006), 572-618. MR 2007f:16033 Zbl 1127.16011

[Caldero and Chapoton 2006] P. Caldero and F. Chapoton, "Cluster algebras as Hall algebras of quiver representations”, Comment. Math. Helv. 81:3 (2006), 595-616. MR 2008b:16015 Zbl 1119. 16013 
[Caldero and Keller 2006] P. Caldero and B. Keller, "From triangulated categories to cluster algebras, II”, Ann. Sci. École Norm. Sup. (4) 39:6 (2006), 983-1009. MR 2008m:16031 Zbl 1115. 18301

[Caldero and Zelevinsky 2006] P. Caldero and A. Zelevinsky, "Laurent expansions in cluster algebras via quiver representations”, Mosc. Math. J. 6:3 (2006), 411-429, 587. MR 2008j:16045 Zbl 1133.16012

[Caldero et al. 2006] P. Caldero, F. Chapoton, and R. Schiffler, "Quivers with relations arising from clusters ( $A_{n}$ case)", Trans. Amer. Math. Soc. 358:3 (2006), 1347-1364. MR 2007a:16025 Zbl 1137.16020

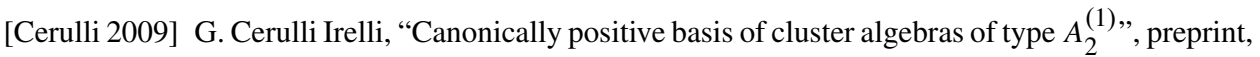
2009. arXiv 0904.2543v2

[Ding et al. 2009] M. Ding, J. Xiao, and F. Xu, "Integral bases of cluster algebras and representations of tame quivers", preprint, 2009. arXiv 0901.1937v1

[Dupont 2008] G. Dupont, "Generic variables in acyclic cluster algebras", preprint, 2008. arXiv $0811.2909 \mathrm{v} 1$

[Dupont 2009] G. Dupont, "Cluster multiplication in regular components via generalized Chebyshev polynomials", preprint, 2009. arXiv 0801.3964v2

[Dupont 2010] G. Dupont, "Quantized Chebyshev polynomials and cluster characters with coefficients”, J. Algebraic Combin. 31:4 (2010), 501-532.

[Fomin and Zelevinsky 2002] S. Fomin and A. Zelevinsky, "Cluster algebras, I: Foundations", J. Amer. Math. Soc. 15:2 (2002), 497-529. MR 2003f:16050 Zbl 1021.16017

[Fomin and Zelevinsky 2003] S. Fomin and A. Zelevinsky, "Cluster algebras, II: Finite type classification”, Invent. Math. 154:1 (2003), 63-121. MR 2004m:17011 Zbl 1054.17024

[Fomin and Zelevinsky 2007] S. Fomin and A. Zelevinsky, "Cluster algebras, IV: Coefficients", Compos. Math. 143:1 (2007), 112-164. MR 2008d:16049 Zbl 1127.16023

[Keller 2005] B. Keller, “On triangulated orbit categories”, Doc. Math. 10 (2005), 551-581. MR 2007c :18006 Zbl 1086.18006

[Ringel 1984] C. M. Ringel, Tame algebras and integral quadratic forms, Lecture Notes in Mathematics 1099, Springer, Berlin, 1984. MR 87f:16027 Zbl 0546.16013

[Sherman and Zelevinsky 2004] P. Sherman and A. Zelevinsky, "Positivity and canonical bases in rank 2 cluster algebras of finite and affine types", Mosc. Math. J. 4:4 (2004), 947-974, 982. MR 2006c:16052 Zbl 1103.16018

[Simson and Skowroński 2007] D. Simson and A. Skowroński, Elements of the representation theory of associative algebras, vol. II, London Mathematical Society Student Texts 71, Cambridge University Press, 2007. MR 2009f:16001 Zbl 1129.16001

[Yang and Zelevinsky 2008] S.-W. Yang and A. Zelevinsky, "Cluster algebras of finite type via Coxeter elements and principal minors", Transform. Groups 13:3-4 (2008), 855-895. MR 2009j:13029 Zbl 1177.16010

Communicated by Andrei Zelevinsky

Received 2009-10-26 Revised 2010-03-17 Accepted 2010-04-16

gregoire.dupont@usherbrooke.ca

Université de Sherbrooke, Département de Mathématiques, 2500 Boulevard de l'université, Sherbrooke J1K 2R1, Canada http://pages.usherbrooke.ca/gdupont2 


\section{Algebra \& Number Theory}

www.jant.org

\section{EDITORS}

\section{MANAGING EDITOR}

Bjorn Poonen

Massachusetts Institute of Technology

Cambridge, USA

\author{
EDITORIAL BOARD CHAIR \\ David Eisenbud \\ University of California \\ Berkeley, USA
}

\section{BOARD OF EDITORS}

Georgia Benkart

Dave Benson

Richard E. Borcherds

John H. Coates

J-L. Colliot-Thélène

Brian D. Conrad

Hélène Esnault

Hubert Flenner

Edward Frenkel

Andrew Granville

Joseph Gubeladze

Ehud Hrushovski

Craig Huneke

Mikhail Kapranov

Yujiro Kawamata

János Kollár

Hendrik W. Lenstra

Yuri Manin

Barry Mazur
University of Wisconsin, Madison, USA

University of Aberdeen, Scotland

University of California, Berkeley, USA

University of Cambridge, UK

CNRS, Université Paris-Sud, France

University of Michigan, USA

Universität Duisburg-Essen, Germany

Ruhr-Universität, Germany

University of California, Berkeley, USA

Université de Montréal, Canada

San Francisco State University, USA

Hebrew University, Israel

University of Kansas, USA

Yale University, USA

University of Tokyo, Japan

Princeton University, USA

Universiteit Leiden, The Netherlands

Northwestern University, USA

Harvard University, USA
Susan Montgomery

Shigefumi Mori

Andrei Okounkov

Raman Parimala

Victor Reiner

Karl Rubin

Peter Sarnak

Michael Singer

Ronald Solomon

Vasudevan Srinivas

J. Toby Stafford

Bernd Sturmfels

Richard Taylor

Ravi Vakil

Michel van den Bergh

Marie-France Vignéras

Kei-Ichi Watanabe

Andrei Zelevinsky

Efim Zelmanov
University of Southern California, USA

RIMS, Kyoto University, Japan

Princeton University, USA

Emory University, USA

University of Minnesota, USA

University of California, Irvine, USA

Princeton University, USA

North Carolina State University, USA

Ohio State University, USA

Tata Inst. of Fund. Research, India

University of Michigan, USA

University of California, Berkeley, USA

Harvard University, USA

Stanford University, USA

Hasselt University, Belgium

Université Paris VII, France

Nihon University, Japan

Northeastern University, USA

University of California, San Diego, USA

\section{PRODUCTION}

ant@mathscipub.org

Silvio Levy, Scientific Editor

Andrew Levy, Production Editor

See inside back cover or www.jant.org for submission instructions.

The subscription price for 2010 is US \$140/year for the electronic version, and \$200/year (+\$30 shipping outside the US) for print and electronic. Subscriptions, requests for back issues from the last three years and changes of subscribers address should be sent to Mathematical Sciences Publishers, Department of Mathematics, University of California, Berkeley, CA 94720-3840, USA.

Algebra \& Number Theory (ISSN 1937-0652) at Mathematical Sciences Publishers, Department of Mathematics, University of California, Berkeley, CA 94720-3840 is published continuously online. Periodical rate postage paid at Berkeley, CA 94704, and additional mailing offices.

ANT peer review and production are managed by EditFLOW ${ }^{\mathrm{TM}}$ from Mathematical Sciences Publishers.

PUBLISHED BY

mathematical sciences publishers

http://www.mathscipub.org

A NON-PROFIT CORPORATION

Typeset in LATEX

Copyright $\odot 2010$ by Mathematical Sciences Publishers 


\section{Algebra \& Number Theory}

Volume $4 \quad$ No. $5 \quad 2010$

On the Spiegelungssatz for the 4-rank

ÉTIENNE FOUVRY and JÜRGEN KLÜNERS

The Manin constant of elliptic curves over function fields AMBRUS PÁL

Le problème de Bogomolov effectif sur les variétés abéliennes

AURÉLIEN GALATEAU

Transverse quiver Grassmannians and bases in affine cluster algebras GRÉGOIRE DUPONT

Connected gradings and the fundamental group 